\title{
The formation of the W43 complex: constraining its atomic-to-molecular transition and searching for colliding clouds
}

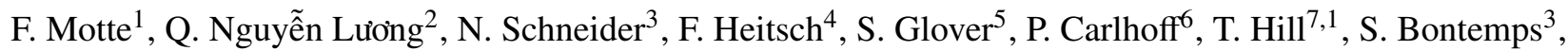 \\ P. Schilke ${ }^{6}$, F. Louvet ${ }^{1}$, M. Hennemann ${ }^{8}$, P. Didelon ${ }^{1}$, and H. Beuther ${ }^{8}$ \\ ${ }^{1}$ Laboratoire AIM Paris-Saclay, CEA/IRFU - CNRS/INSU - Université Paris Diderot, Service d'Astrophysique, Bât. 709, \\ CEA-Saclay, 91191 Gif-sur-Yvette Cedex, France \\ e-mail: frederique.motte@cea. fr \\ 2 Canadian Institute for Theoretical Astrophysics, University of Toronto, 60 St. George Street, Toronto, ON M5S 3H8, Canada \\ 3 OASU/LAB-UMR 5804, CNRS/INSU - Université Bordeaux 1, 2 rue de l'Observatoire, BP 89, 33270 Floirac, France \\ 4 Department of Physics and Astronomy, University of North Carolina Chapel Hill, Phillips Hall, Chapel Hill, NC 27599-3255, USA \\ 5 Universität Heidelberg, Zentrum für Astronomie, Institut für Theoretische Astrophysik, Albert-Ueberle-Str. 2, 69120 Heidelberg, \\ Germany \\ ${ }^{6}$ Physikalisches Institut, Universität zu Köln, Zülpicher Str. 77, 50937 Köln, Germany \\ 7 Joint ALMA Observatory, 3107 Alonso de Cordova, Vitacura, Santiago, Chile \\ 8 Max-Planck-Institut für Astronomie, Königsstuhl 17, 69117 Heidelberg, Germany
}

Received 7 November 2013 / Accepted 17 April 2014

\begin{abstract}
Context. Numerical simulations have explored the possibility of forming molecular clouds through either a quasi-static, selfgravitating mechanism or the collision of gas streams or lower density clouds. They also quantitatively predict the distribution of matter at the transition from atomic to molecular gases.

Aims. We aim to observationally test these models by studying the environment of W43, a molecular cloud complex recently identified near the tip of the Galactic long bar.

Methods. Using Galaxy-wide H I and ${ }^{12} \mathrm{CO} 1-0$ surveys, we searched for gas flowing toward the W43 molecular cloud complex. We also estimated the $\mathrm{HI}$ and $\mathrm{H}_{2}$ mass surface densities to constrain the transition from atomic to molecular gas around and within W43. Results. We found three cloud ensembles within the position-velocity diagrams of ${ }^{12} \mathrm{CO}$ and $\mathrm{HI}$ gases. They are separated by $\sim 20 \mathrm{~km} \mathrm{~s}^{-1}$ along the line of sight and extend into the ${ }^{13} \mathrm{CO}$ velocity structure of W43. Since their velocity gradients are consistent with free fall, they could be nearby clouds attracted by and streaming toward the W43 $\sim 10^{7} M_{\odot}$ potential well. We show that the H I surface density, $\Sigma_{\mathrm{HI}}=45-85 M_{\odot} \mathrm{pc}^{-2}$, does not reach any threshold level but increases when entering the $130 \mathrm{pc}$-wide molecular complex previously defined. This suggests that an equilibrium between $\mathrm{H}_{2}$ formation and photodissociation has not yet been reached. The $\mathrm{H}_{2}$-to-H I ratio measured over the W43 region and its surroundings, $R_{\mathrm{H}_{2}} \sim 3.5 \pm_{2}^{3}$, is high, indicating that most of the gas is already in molecular form in W43 and in structures several hundred parsecs downstream along the Scutum-Centaurus arm. Conclusions. The W43 molecular cloud complex may have formed and, in fact may still be accreting mass from the agglomeration of clouds. Already in the molecular-dominated regime, most of these clouds are streaming from the Scutum-Centaurus arm. This clearly disagrees with quasi-static and steady-state models of molecular cloud formation.
\end{abstract}

Key words. ISM: clouds - ISM: structure - stars: formation - stars: massive - ISM: atoms - evolution

\section{Introduction}

Although applicable to all mechanisms of cloud formation, the term "converging flows" generally refers to the convergence of H I streams that can naturally be driven by local instabilities in the disk, such as those due to gravity, supernova explosions, or spiral shocks (e.g., Koyama \& Inutsuka 2000; Heitsch et al. 2005; Vázquez-Semadeni et al. 2007). For the past decade, numerical models have investigated the capability of such colliding flows (warm neutral medium, $\sim 6000 \mathrm{~K}$ ) to form cold structures (cold neutral medium, $\sim 70 \mathrm{~K}$ ) through shocks (e.g., Vázquez-Semadeni et al. 1996; Hennebelle \& Pérault 1999; Ballesteros-Paredes et al. 1999). However, it is only recently that 3D models have simulated the thermal transition from the atomic to the molecular phase $\left(\mathrm{H} \mathrm{I} \rightarrow \mathrm{H}_{2}\right)$ with realistic heating and cooling functions (e.g., Audit \& Hennebelle 2005; Glover $\&$ Mac Low 2007). Several groups aim at studying in detail the formation of molecular clouds with high enough resolution to model star formation. Recent 3D numerical simulations now include gravity, magnetic fields, and thermal and dynamical instabilities (e.g. Banerjee et al. 2009; Hartmann et al. 2012) and in some cases treat the chemical evolution of the gas (Heitsch \& Hartmann 2008; Clark et al. 2012; Inoue \& Inutsuka 2012).

Massive giant molecular clouds or cloud complexes may alternatively form from the collision and/or agglomeration of clouds (e.g., Blitz \& Shu 1980; Dobbs 2008). Clouds are higher density and more structured media than diffuse $\mathrm{H}$ I streams, and so they are mainly composed of cold $\mathrm{HI}$ and $\mathrm{H}_{2}$ gas. Cloudcloud collisions are expected to be the dominant phenomenon in high-density regions of galaxies (e.g., Dobbs 2008) and during the late-stage evolution of molecular clouds formed through $\mathrm{H} \mathrm{I}$ converging flows (e.g., Vázquez-Semadeni et al. 2010). Several groups are studying the formation, evolution, and disruption 
of massive clouds by spiral waves, bar potentials, and satellite galaxies with hydrodynamic simulations of galaxies able to resolve molecular clouds (e.g., Bournaud et al. 2010; Dobbs \& Pringle 2013).

Both colliding flows and cloud-cloud collisions naturally explain the observed rapid onset of star formation once the cloud has formed, which has proven problematic in the past $\left(<3-10 \times 10^{6} \mathrm{yr}\right.$, Ballesteros-Paredes et al. 1999; Hartmann et al. 2001; Roman-Duval et al. 2009). In these scenarios, molecular clouds are never in a kinematic equilibrium state, because part of the cloud collapses while part of it disperses. A few models have described the formation of molecular clouds by assuming equilibrium between the formation of $\mathrm{H}_{2}$ molecules and their photodissociation (e.g., Andersson \& Wannier 1993; Krumholz et al. 2009). In short, these authors applied a simplified version of the chemical modeling of photodissociation regions (e.g., van Dishoeck \& Black 1986) to a spherical geometry. However, such an equilibrium may never be reached since its timescale should be $1-3 \times 10^{7}$ yr or even longer (Mac Low \& Glover 2012). Non steady-state models have been developed using turbulence and/or colliding streams to enhance and accelerate the formation of $\mathrm{H}_{2}$ molecules behind shocks (e.g., Heitsch et al. 2008; Hennebelle et al. 2008; Koyama \& Inutsuka 2000; Clark et al. 2012).

These two families of models make different predictions for the conversion of $\mathrm{HI}$ into $\mathrm{H}_{2}$, especially in terms of the lifetime of the process and the mixing of the $\mathrm{H}_{2}$ and $\mathrm{HI}$ gases. Indeed, steady-state models assume that an equilibrium between the $\mathrm{H} \mathrm{I}$ and $\mathrm{H}_{2}$ formation and destruction is rapidly reached. The $\mathrm{H} \mathrm{I}$ and $\mathrm{H}_{2}$ gases are also assumed to be mutually exclusive, with a sharp transition from $\mathrm{H}$ I to $\mathrm{H}_{2}$-dominated media when entering the cloud (e.g., Krumholz et al. 2009). As a consequence, equilibrium models predict the existence of a threshold for the atomic gas surface density, at $\Sigma_{\mathrm{HI}} \simeq 10 M_{\odot} \mathrm{pc}^{-2}$ for solar metallicity gas according to Krumholz et al. (2009). In contrast, non-steady state models start with an accumulation of $\mathrm{H} \mathrm{I}$ gas that translates into a mass surface density above this classical threshold. The $\mathrm{H}_{2}$ formation rate, high at the beginning of the molecular cloud formation process, continuously slows down as the cloud becomes fully molecular (e.g., Clark et al. 2012). The H I surface density thus decreases over time, first sharply, then slower and slower, eventually reaching this equilibrium state.

W43 should be a perfect testbed to investigate the formation of molecular clouds and star formation in the framework of dynamical scenarios. At only $5.5 \mathrm{kpc}$ from us, W43 is among the most extreme molecular cloud complexes of the Milky Way (Nguyen Luong et al. 2011b; Zhang et al. 2014). It is massive, $M_{\text {total }} \sim 6 \times 10^{6} M_{\odot}^{1}$, within an equivalent diameter of $\sim 130 \mathrm{pc}$, and is highly concentrated into dense star-forming sites (Nguyen Luong et al. 2011b). Despite a velocity dispersion FWHM of $\sim 22.3 \mathrm{~km} \mathrm{~s}^{-1}$, the W43 molecular cloud complex is a coherent and gravitationally bound ensemble of clouds. Moreover, W43 has the potential to form starburst clusters in the near future (SFR $\sim 0.1 M_{\odot} \mathrm{yr}^{-1}$, Nguyen Luong et al. 2011b; Louvet et al. 2014). Its densest parts correspond to the Galactic mini-starburst cloud W43-Main (Motte et al. 2003) recently mapped by the Herschel/Hi-GAL and HOBYS key programs (Molinari et al. 2010; Motte et al. 2010). Nguyen-Luong et al. (2013) identified two dense filamentary clouds within W43-Main categorized as ridges following the definition of Hill et al. (2011) and

\footnotetext{
1 The mass and diameter values given in Nguyen Luong et al. (2011b) have been recalculated to account for the refinement of W43 distance, from $6 \mathrm{kpc}$ to $5.5 \mathrm{kpc}$, by Zhang et al. (2014).
}

Table 1. Main observational parameters.

\begin{tabular}{ccccc}
\hline \hline $\begin{array}{c}\text { Survey } \\
/ \text { tracer }\end{array}$ & $\begin{array}{c}\text { Frequency } \\
{[\mathrm{GHz}]}\end{array}$ & $\begin{array}{c}H P B W \\
{\left[{ }^{\prime \prime}\right]}\end{array}$ & $\begin{array}{c}\Delta v_{\text {res }} \\
{\left[\mathrm{km} \mathrm{s}^{-1}\right]}\end{array}$ & $\begin{array}{c}1 \sigma \mathrm{rms} \\
{\left[\mathrm{K} \mathrm{km} \mathrm{s}^{-1}\right]}\end{array}$ \\
\hline $\mathrm{VGPS} / \mathrm{H} \mathrm{I}$ & 1.420 & 60 & 1.56 & 1.80 \\
$\mathrm{CfA} /{ }^{12} \mathrm{CO} 1-0$ & 115.271 & 450 & 0.65 & 0.22 \\
\hline
\end{tabular}

Hennemann et al. (2012). In short, ridges are elongated clouds with very high column density, $N_{\mathrm{H}_{2}}>10^{23} \mathrm{~cm}^{-2}$, over several squared parsecs (see also Nguyen Luong et al. 2011a; Motte et al. 2012). Nguyen-Luong et al. (2013) proposed and Louvet et al. (2014) showed that the W43-MM1 and W43-MM2 ridges are progenitors of young massive clusters. W43 is located near the meeting point of the Scutum-Centaurus (or Scutum-Crux) Galactic arm and the Galactic long bar, a dynamically complex region where collisions are expected (Nguyen Luong et al. 2011b; Carlhoff et al. 2013). Several massive stellar associations, called red supergiant clusters, have also been identified in the $1^{\circ}-6^{\circ}$ neighborhood of W43 (see González-Fernández et al. 2012, and references therein).

In this paper, we attempt to trace back the formation of molecular clouds in the W43 complex. Employing the database described in Sect. 2, we look for gas streams and cloud ensembles at the outskirts of the atomic and molecular complex and characterize its atomic envelope (see Sect. 3). Section 4 follows the transition from atomic to molecular media and discusses the collision probability of gas streams and clouds. Finally, Sect. 5 concludes that the equilibrium relations of the $\mathrm{HI}$ into $\mathrm{H}_{2}$ transition do not apply in W43 and that this extreme cloud complex may have been created by the collision of molecular clouds.

\section{Observations}

The atomic gas data for W43 are taken from the Very Large Array (VLA) Galactic Plane Survey (VGPS ${ }^{2}$, Stil et al. 2006). VGPS is a survey of the $21 \mathrm{~cm}$ continuum and line emission from neutral atomic hydrogen, H I, performed throughout the Galactic plane $\left(18^{\circ}<l<67^{\circ},|b|<1.3^{\circ}\right)$ by the VLA and combined with short-spacing information obtained with the Green Bank Telescope (GBT). This data set has a spatial resolution of $1^{\prime} \times 1^{\prime}$ and a velocity resolution of $1.56 \mathrm{~km} \mathrm{~s}^{-1}$ spanning the range -120 to $170 \mathrm{~km} \mathrm{~s}^{-1}$.

To trace the low-density molecular gas, we used the ${ }^{12} \mathrm{CO} 1-0$ data from the Galactic plane survey made with the CfA 1.2 m telescope $^{3}$ (Dame et al. 2001). These data have a spatial resolution of $450^{\prime \prime}$ and a velocity resolution of $0.65 \mathrm{~km} \mathrm{~s}^{-1}$ spanning the velocity range -0.5 to $271 \mathrm{~km} \mathrm{~s}^{-1}$. Note that ${ }^{13} \mathrm{CO}$ data of e.g. the GRS survey (Jackson et al. 2006) trace higher density gas than ${ }^{12} \mathrm{CO}$ and are thus less relevant for our study of the transition from atomic to molecular gas. The main characteristics of the data sets used here are listed in Table 1.

Low-density cloud structures surrounding the W43 complex as defined by Nguyen Luong et al. (2011b) are expected to display optically thin H I and ${ }^{12} \mathrm{CO}$ lines. In contrast, these two lines should be self-absorbed toward the densest parts of W43. H I lines will also be absorbed by the continuum free-free

2 The full spectral cubes of the VGPS database are available as FITS files at http://wWw.ras.ucalgary.ca/VGPS/. The VLA and GBT are facilities of the National Radio Astronomy Observatory.

3 The CfA telescope is the $1.2 \mathrm{~mm}$ Millimeter-Wave Telescope at the Center for Astrophysics, Harvard University. 


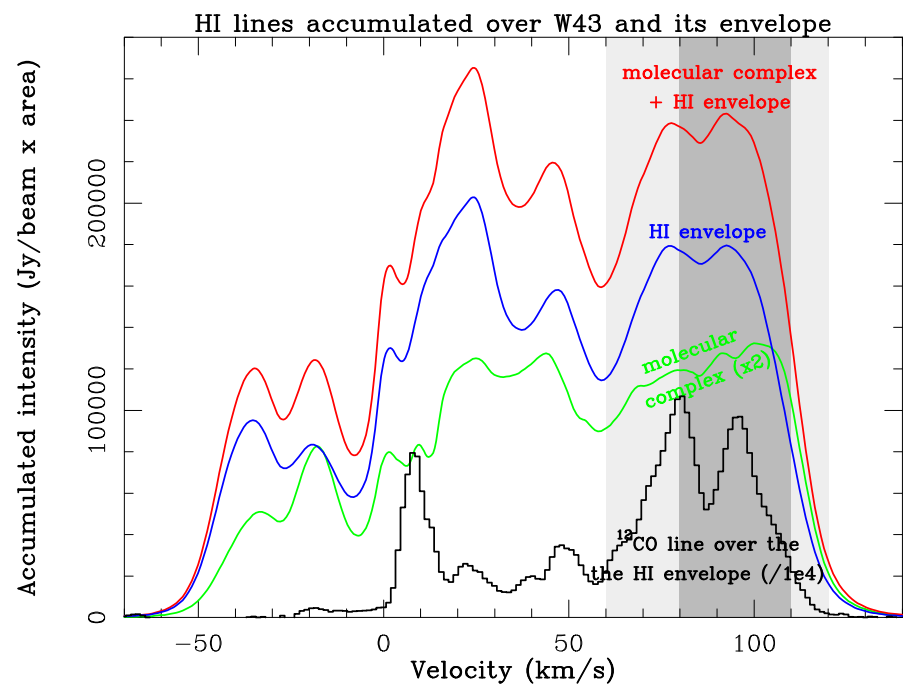

Fig. 1. W43 H I lines (curves) delimited well by the $60-120 \mathrm{~km} \mathrm{~s}^{-1}$ velocity range and displaying a double-peak shape similar to the ${ }^{12} \mathrm{CO} 1-0$ lines (black histogram). H I spectra result from the sum over all observed beams in the $1.8^{\circ} \times 0.8^{\circ}$ area covering the $\mathrm{W} 43$ molecular complex (green curve, $\times 2$, within the white rectangle of Fig. 2), those within its $\mathrm{HI}$ envelope (blue curve, in between the white continuous and dashed rectangles of Fig. 2) and the $3^{\circ} \times 2^{\circ}$ area covering both components (red curve, within the white dashed rectangle of Fig. 2). The main and complete velocity ranges of W43 defined by Nguyen Luong et al. (2011b) are indicated by the light and dark gray shaded regions, respectively.

emission of H II regions, seen as pointlike sources within the densest parts of W43.

\section{Results and analysis}

Here we discuss the velocity range covered by the ${ }^{12} \mathrm{CO}$ and $\mathrm{H} \mathrm{I}$ envelope of W43 (see Sect. 3.1), characterize the H I envelope of W43 (see Sect. 3.2), and search for signatures of colliding flows or clouds (see Sect. 3.3).

\subsection{Velocity range of the ${ }^{12} \mathrm{CO} / \mathrm{H}$ I envelope of $\mathrm{W} 43$}

Figure 1 displays the shape of the $\mathrm{H}$ I lines of Table 1 summed over areas covering the W43 molecular complex, its ${ }^{12} \mathrm{CO} / \mathrm{H} \mathrm{I}$ envelope, and the combination of the two. According to Nguyen Luong et al. (2011b), the W43 molecular cloud complex extends in Galactic longitude from $l=29.6^{\circ}$ to $31.4^{\circ}$ and in Galactic latitude from $b=-0.5^{\circ}$ to $b=0.3^{\circ}$. If the ${ }^{12} \mathrm{CO} / \mathrm{H}$ I envelope of the complex is also included, the combined region extends from $l=29^{\circ}$ to $32^{\circ}$ and $|b|<1^{\circ}$. The corresponding areas are outlined in Fig. 2, and lines integrated over these areas are displayed in Fig. 1. Comparison of the $\mathrm{HI}$ and ${ }^{12} \mathrm{CO}$ spectra shown in Fig. 1 with the ${ }^{13} \mathrm{CO}$ spectra discussed by Carlhoff et al. (2013) situates the $0-60 \mathrm{~km} \mathrm{~s}^{-1}$ velocity components in the foreground of W43 (mostly within the Sagittarius arm) and the $-70-0 \mathrm{~km} \mathrm{~s}^{-1}$ components in its background. A complete disentangling of $\mathrm{CO}$ velocity components in the foreground and background of W43 can be found in Carlhoff et al. (2013, see especially their Fig. 5).

In Fig. 1, the ${ }^{12} \mathrm{CO}$ and $\mathrm{H}$ I lines of Table 1, observed toward W43 and its surrounding envelope are broad and structured. They happen to be delimited fairly well by the 60 and $120 \mathrm{~km} \mathrm{~s}^{-1}$ velocities, marking out the so-called "complete velocity range" of $\mathrm{W} 43$. We recall that two velocity ranges have been defined for W43 using ${ }^{13} \mathrm{CO} 1-0$ data from the GRS survey
(Jackson et al. 2006) and the higher resolution ${ }^{13} \mathrm{CO} 2-1 \mathrm{im}-$ age of the W43-HERO ${ }^{4}$ large program (Nguyen Luong et al. $2011 \mathrm{~b}$; Carlhoff et al. 2013). The main velocity range $\left(v_{\mathrm{LSR}}=\right.$ $80-110 \mathrm{~km} \mathrm{~s}^{-1}$ ) traces the central part of the ${ }^{13} \mathrm{CO}$ cloud, while the complete range $\left(60-120 \mathrm{~km} \mathrm{~s}^{-1}\right)$ also includes peripheral lower density clouds. Since the $\mathrm{HI}$ and ${ }^{12} \mathrm{CO} 1-0$ lines trace lower density gas than ${ }^{13} \mathrm{CO}$, the velocity range over which they are detected is generally broader.

The Hi lines of the envelope surrounding W43 sum up in the $60-120 \mathrm{~km} \mathrm{~s}^{-1}$ range of Fig. 1 into two main velocity components, leading to a combined FWHM $\sim 60 \mathrm{~km} \mathrm{~s}^{-1}$. These two velocity peaks are also observed in the ${ }^{12} \mathrm{CO} 1-0$ line arising from the envelope of W43 and so do not appear to be due to H I self-absorption. Indeed, the shape of $\mathrm{HI}$ and $\mathrm{CO}$ lines at the highest positive and negative latitudes of the VGPS and CfA data, at $|b|>1^{\circ}$, are as complicated as the spectra displayed in Fig 1 . The variation of the line shapes when entering the envelope of W43 or W43 itself also do not suggest that they gradually become optically thick. These line shapes provide some evidence that the H I line is optically thin throughout the envelope of W43 and that at least some of the emission from the W43 complex itself is also optically thin. However, this still needs to be confirmed, and will be quantified using a higher resolution H I emission map with the VLA, as well as H I absorption measurements toward radio sources (Bihr et al., in prep.).

Assuming that the H I line shape observed for the H I envelope also applies to the W43 molecular complex, one can make a crude estimate of the optical thickness of H I in W43. The lines observed in Fig. 1 display a similar shape over a wide range of velocities, suggesting that the total H I flux integrated over the $1.8^{\circ} \times 0.8^{\circ}$ area of W43 is globally underestimated, but by no more than $20 \%$. Section 3.2 and Fig. 7 more precisely indicate the extent of the W43 central region where the H I line is largely optically thick. Given the above results, we interpret the complicated shape of the $\mathrm{HI}$ and ${ }^{12} \mathrm{CO}$ lines of Fig. 1 as reflecting the complex velocity pattern of the W43 region rather than optical thickness. As further discussed in Sect. 3.3 and illustrated in Figs. 3-5, the two velocity peaks observed in Fig. 1 correspond to ${ }^{12} \mathrm{CO} / \mathrm{H}$ I cloud ensembles at different velocities.

In the following, we integrate all lines over the $60-120 \mathrm{~km} \mathrm{~s}^{-1}$ velocity range. With a narrower integration range, the velocity investigations and comparison of the $\mathrm{HI}$ and $\mathrm{H}_{2}$ surface densities made in Sects. 3.3 and 4 are qualitatively unchanged. As for the absolute values of the ${ }^{12} \mathrm{CO}$ and $\mathrm{HI}$ integrated intensities and thus the $\mathrm{H}_{2}$ and $\mathrm{H}$ I gas masses, they only decrease by $\sim 30 \%$ and $\sim 40 \%$, respectively, for a $80-110 \mathrm{~km} \mathrm{~s}^{-1}$ integration range.

\subsection{A clear HI envelope around W43}

Figure 2 displays the ${ }^{12} \mathrm{CO} 1-0$ and $\mathrm{HI}$ images of the W43 molecular complex and its surroundings, integrated over $v_{\mathrm{LSR}}=$ $60-120 \mathrm{~km} \mathrm{~s}^{-1}$. The observed $\mathrm{HI}$ and ${ }^{12} \mathrm{CO}$ structures correlate quite well along the Galactic plane, out to the outskirts of the W43 complex, which is outlined by a white rectangle: roughly at $l>31^{\circ}$ and $l<29.75^{\circ}$. Conversely, the $\mathrm{H}$ and ${ }^{12} \mathrm{CO}$ emissions are anticorrelated in the densest parts of the molecular complex. This corresponds to areas where the ${ }^{12} \mathrm{CO} 1-0$ brightness increases from $140 \mathrm{~K} \mathrm{~km} \mathrm{~s}^{-1}$ to $200 \mathrm{~K} \mathrm{~km} \mathrm{~s}^{-1}$ in

\footnotetext{
4 The W43-HERO (W43 Hera/EmiR Observation) project is an IRAM $30 \mathrm{~m}$ large program, whose description and data can be accessed at http://www.iram-institute.org/EN/ content-page-292-7-158-240-292-0.html
} 


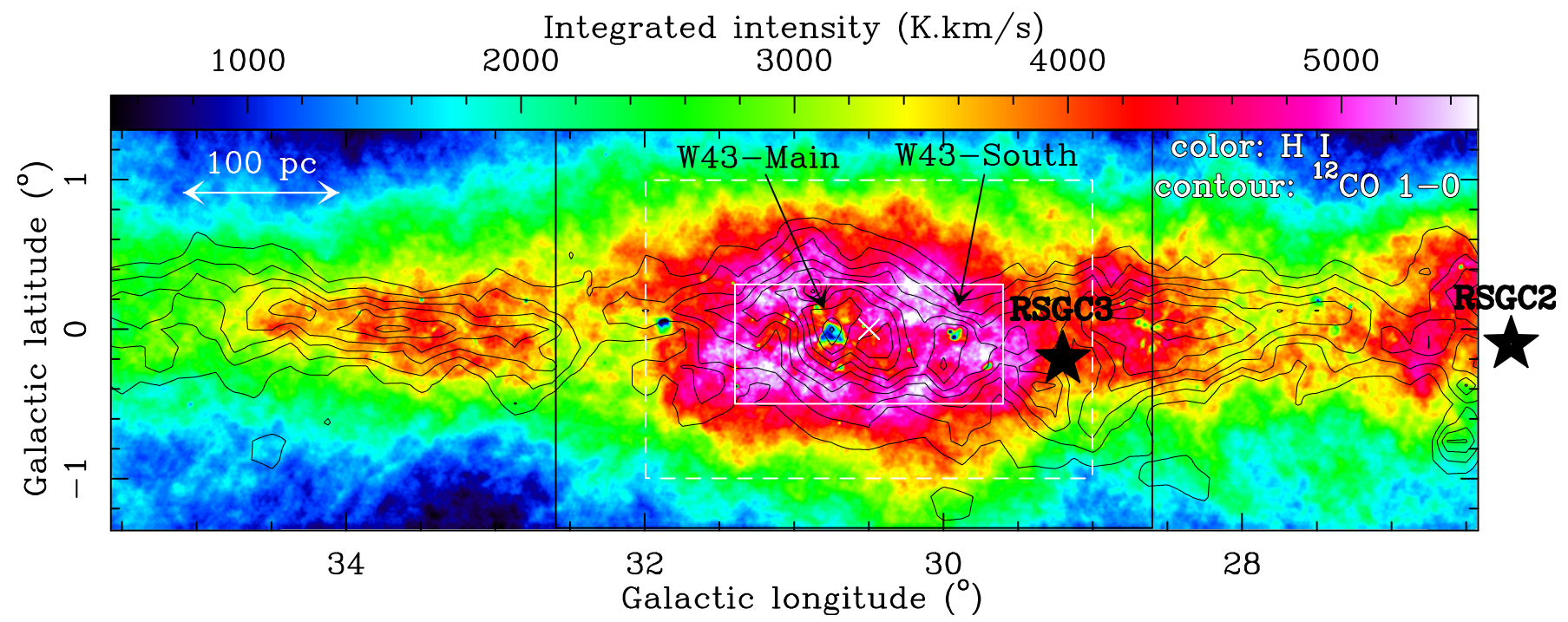

Fig. 2. Galactic plane surroundings of the W43 molecular complex as seen in $\mathrm{H} \mathrm{I}$ (color) and ${ }^{12} \mathrm{CO} 1-0$ (contours) integrated over $60-120 \mathrm{~km} \mathrm{~s}^{-1}$, the complete velocity range of W43. The contours go from 40 to $200 \mathrm{~K} \mathrm{~km} \mathrm{~s}^{-1}$ with a step of $20 \mathrm{~K} \mathrm{~km} \mathrm{~s}^{-1}$, and are always monotonously increasing, never displaying ${ }^{12} \mathrm{CO}$ dips (see Fig. 6b). The white boxes outline the extent of the W43 molecular complex (continuous lines) and its H I envelope (dashed lines) as defined in Nguyen Luong et al. (2011b). The black box locates the largest ring used in the $R_{\mathrm{H}_{2}}$ diagram (see Fig. 7) and the white cross its center. Stars pinpoint the red supergiant clusters/associations (RSGC2 and RSGC3) identified in the neighborhood.

Fig. 2 (i.e. within the seventh contour) while the HI emission decreases from $\sim 6000 \mathrm{~K} \mathrm{~km} \mathrm{~s}^{-1}$ (pink ring in Fig. 2) down to $\sim 1000 \mathrm{~K} \mathrm{~km} \mathrm{~s}^{-1}$ (blue pixels toward W43-Main). Figure 2 also confirms that the ${ }^{12} \mathrm{CO}$ and $\mathrm{HI}$ gases are confined by the midplane pressure of the Milky Way with a width at half maximum of about $0.8^{\circ}$ and $2^{\circ}$, respectively.

Observationally, $\mathrm{H}_{2}$ and $\mathrm{HI}$ are known to be intimately linked on all scales, from those of large molecular complexes (e.g., Blitz \& Thaddeus 1980; Elmegreen \& Elmegreen 1987) to the scales of individual filamentary clouds (e.g., Wannier et al. 1983; Ballesteros-Paredes et al. 1999). Position and velocity coincidences for $\mathrm{HI}$ and $\mathrm{CO}$ have been noted for various clouds and complexes (e.g., Blitz \& Thaddeus 1980; Elmegreen \& Elmegreen 1987). Halos of warm H I gas have been found, generally asymmetrically surrounding molecular structures, in the close outskirts of $10 \mathrm{pc}$ filaments/clouds of the nearby Gould Belt clouds (see Wannier et al. 1983; Ballesteros-Paredes et al. 1999; Lee et al. 2012, and references therein). Similar asymmetrical halos were observed by Williams et al. (1995) for the high-mass star-forming molecular cloud complex Rosette and its filaments.

The H I envelope of the W43 molecular complex itself displays an elliptical shape that symmetrically surrounds the molecular complex (see Fig. 2 of the present paper and Fig. 8 of Nguyen Luong et al. 2011b). This envelope, with a median diameter of $\sim 270 \mathrm{pc}$, is far enough from the internal UV fields of the H II regions or OB clusters associated with W43 to feel little influence from them. They would otherwise distort its shape. It also lies far enough from the center of the complex to avoid regions where the cold HI gas mixed with molecular gas creates H I self-absorption features that could distort the H I envelope emission. Since the H I envelope has its center close to the Galactic plane, the Galactic interstellar radiation field bathing W43 is expected to be rather isotropic and able to create a symmetrical envelope. The powerful radiation field of RSGC3 could itself distort the W43 envelope at low Galactic longitude if it were not located a few hundred parsecs closer to the Galactic center (see Fig. 2 and Sect. 4.5). The H I envelopes of molecular complexes centered on the Galactic plane are not always symmetric (Schneider, priv. com.). The large size and simple geometry of the W43 H I envelope is therefore rare (see, however, Andersson et al. 1992), making W43 an excellent laboratory to track the transition from the atomic to the molecular medium (see Sects. 4.1-4.2). It also suggests that other processes, such as the formation of molecular clouds in the W43 complex, could be invoked to explain its symmetry on large scales. As proposed in Sect. 4.4, gas could accumulate in front of the long bar and collide to form W43, allowing a symmetric distribution of its $\mathrm{HI}$ and $\mathrm{H}_{2}$ gases on hundred parcsec scales. Position offsets between $\mathrm{HI}$ and $\mathrm{H}_{2}$ are present on smaller scales, as mentioned in Sect. 3.3 below.

\subsection{W43, at the meeting point of large-scale cloud ensembles}

We investigated the link between the $\mathrm{H}_{2}$ and $\mathrm{H}$ I gas further in the W43 molecular complex and its surroundings by comparing the ${ }^{12} \mathrm{CO} 1-0 \mathrm{CfA}$ and H I VGPS datacubes. Figures 3, 4 display the position-velocity diagrams of the W43 molecular complex and its ${ }^{12} \mathrm{CO} / \mathrm{H}$ I envelope (area outlined in Fig. 2) plotted against the Galactic latitude and longitude, respectively. According to the definition of Nguyen Luong et al. (2011b), we used longitude and latitude extents of $l=29^{\circ}$ to $32^{\circ}, b= \pm 1^{\circ}$, embracing the ${ }^{12} \mathrm{CO} / \mathrm{H}$ I envelope of the ${ }^{13} \mathrm{CO}$ complex. Figures $5 \mathrm{a}-\mathrm{b}$ complement our investigation of the $\mathrm{HI}$ and ${ }^{12} \mathrm{CO}$ datacubes for two other latitude ranges. As in Sect. 3.2, we integrated the ${ }^{12} \mathrm{CO}$ and $\mathrm{H}$ I lines over the $v_{\mathrm{LSR}}=60-120 \mathrm{~km} \mathrm{~s}^{-1}$ velocity range.

In Fig. 3a, the ${ }^{12} \mathrm{CO}$ emission displays two peaks within the white rectangle covering the W43 complex, one at $\sim 95 \mathrm{~km} \mathrm{~s}^{-1}$ and another at $\sim 78 \mathrm{~km} \mathrm{~s}^{-1}$, recalling the two velocity peaks of Fig. 1. In addition, there is a component of lower density gas, located at a more negative latitude (from $b=-1.4^{\circ}$ to $b=-0.4^{\circ}$ ) and at $\sim 75 \pm 10 \mathrm{~km} \mathrm{~s}^{-1}$. This component remains prominent in the immediate surroundings of W43 and displays a gradient of $\sim 0.2 \mathrm{~km} \mathrm{~s}^{-1} \mathrm{pc}^{-1}$ over $\sim 40 \mathrm{pc}$ in the direction of increasing Galactic latitude (see Fig. 3b). This component is associated with a ${ }^{12} \mathrm{CO}$ velocity gradient of $\sim 0.1 \mathrm{~km} \mathrm{~s}^{-1} \mathrm{pc}^{-1}$ 
F. Motte et al.: Atomic-to-molecular transition and putative colliding clouds in W43
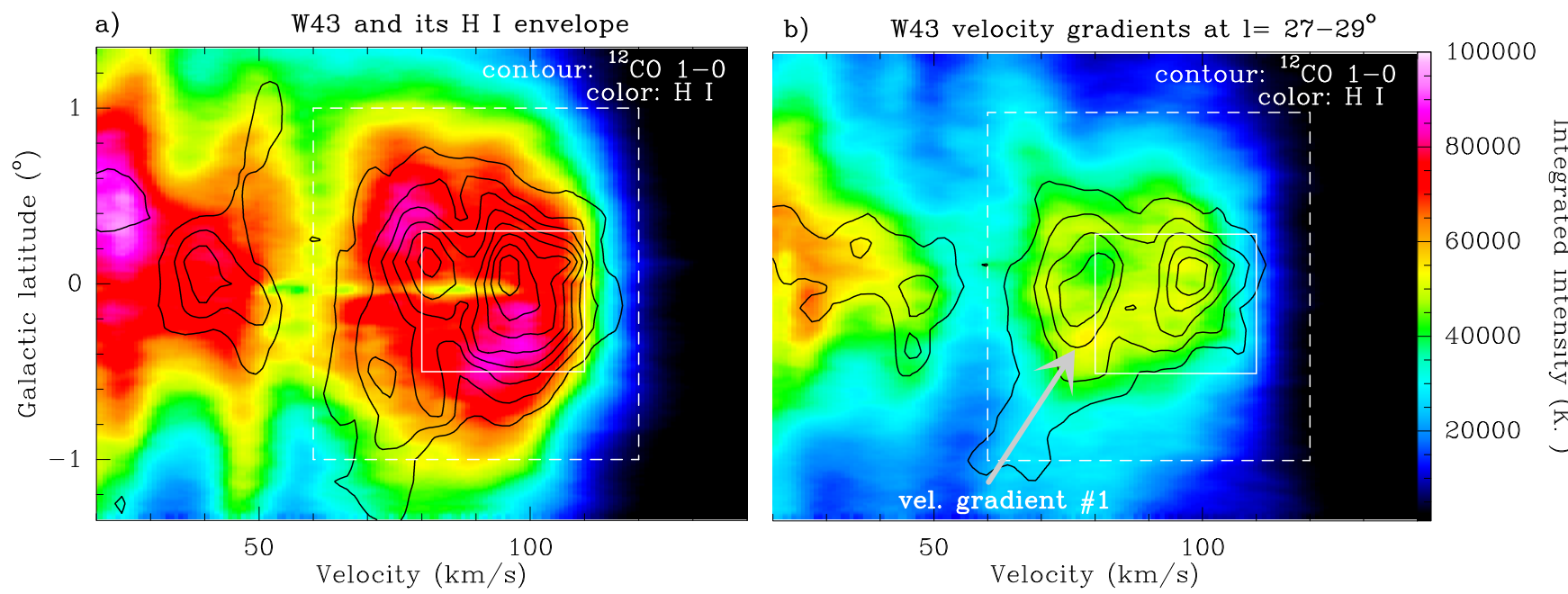

Fig. 3. Position-velocity diagrams confirming the presence of two velocity components in $\mathrm{W} 43$ (see Sect. 3.1 ) and revealing the $\mathrm{HI} /{ }^{12} \mathrm{CO} 1-0$ cloud ensemble \#1 (white arrow, see Sect. 3.3). The distributions of $\mathrm{HI}$ (color) and ${ }^{12} \mathrm{CO} 1-0$ (contours) are plotted against Galactic latitude, kept as the $y$ axis, as in Fig. 2. The H I and ${ }^{12} \mathrm{CO} 1-0$ lines have been summed in a) over the $l=29^{\circ}-32^{\circ}$ longitude range corresponding to W43 and its $\mathrm{HI}$ envelope and in b) over the $l=27^{\circ}-29^{\circ}$ longitude range covering part of the $\mathrm{H} \mathrm{I} /{ }^{12} \mathrm{CO}$ cloud ensembles presented in Sect. 3.3. The contours run from 400 to $2000 \mathrm{~K}$ with a step of $400 \mathrm{~K}$. The white boxes represent the latitude extent and velocity range of the W43 molecular complex (continuous lines) and its $\mathrm{H}$ I envelope (dashed lines).

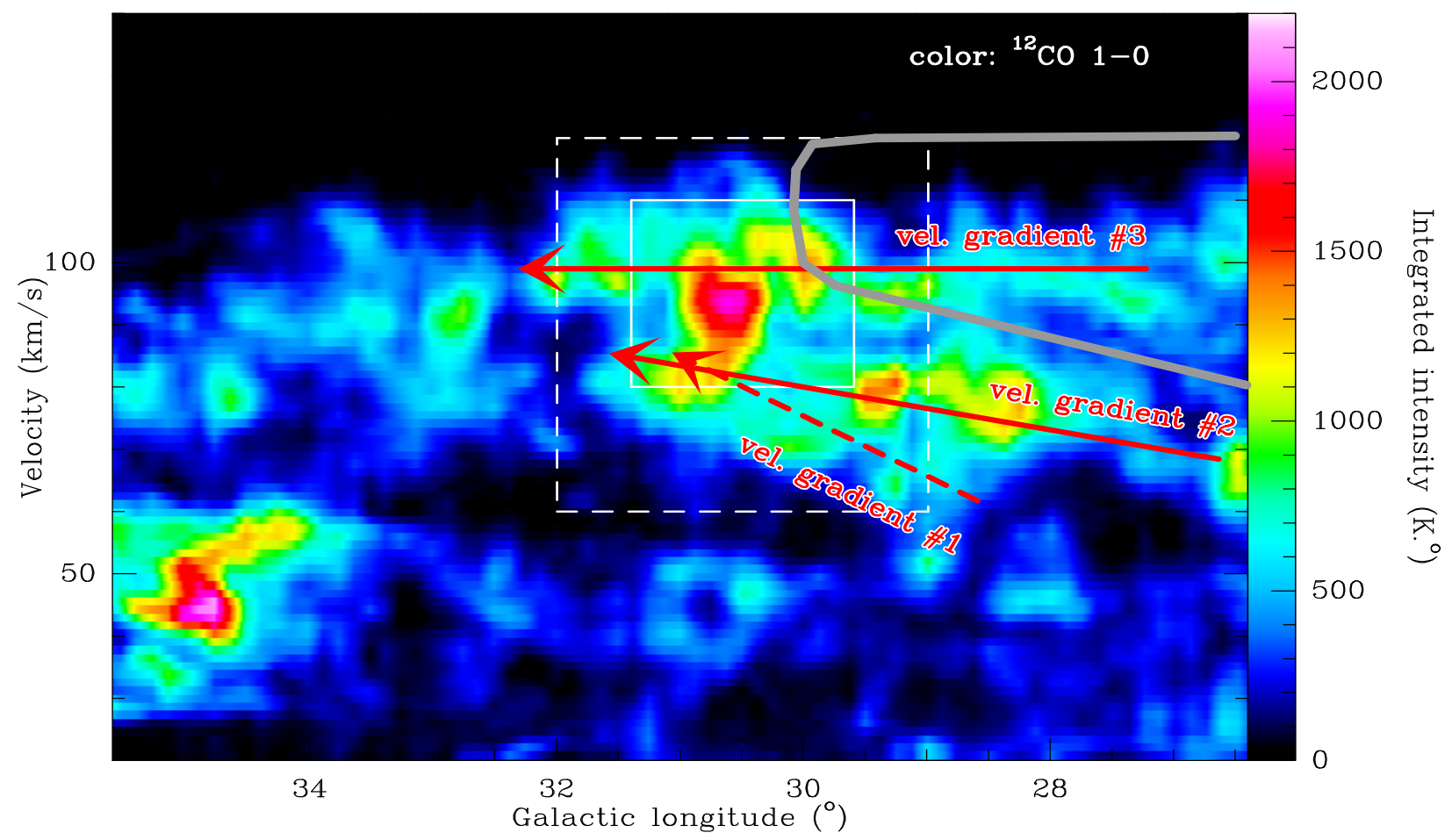

Fig. 4. Position-velocity diagram unfolding the two velocity components of $\mathrm{W} 43$ into the ${ }^{12} \mathrm{CO} 1-0$ cloud ensembles \#2 and \#3 (arrows, see Sect. 3.3). The distributions of ${ }^{12} \mathrm{CO} 1-0$ (color) are plotted against the Galactic longitude, kept as the $x$ axis, as in Fig. 2 . The ${ }^{12} \mathrm{CO} 1-0$ lines have been summed over the $b=-1^{\circ}$ to $b=1^{\circ}$ latitude range corresponding to the W43 complex and its H I envelope. The white boxes represent the longitude extent and velocity range of the W43 molecular complex (continuous lines) and its H I envelope (dashed lines). The thick gray curve indicates the theoretical location of the Scutum-Centaurus arm in the model by Vallée (2008).

over $\sim 400 \mathrm{pc}$ in the direction of increasing longitude (see Fig. $5 \mathrm{a}$ and Fig. 4). It is hereafter labeled as cloud ensemble \#1 (see also Figs. 3-5). When approaching the location of W43, the velocity of cloud ensemble \#1 becomes closer to the main velocity range of $\mathrm{W} 43$. The ${ }^{12} \mathrm{CO}$ dispersion in velocity also becomes narrower (see Figs. 3b and 5a), suggesting that it could be subject to the increasing gravitational influence of W43. This negative latitude component is associated with enhanced $\mathrm{HI}$ emission (see Fig. 3b) though the velocity gradients, suggested by the ${ }^{12} \mathrm{CO}$ position-velocity diagrams, are generally not as clearly defined in the $\mathrm{H}$ I position-velocity diagrams.

Two other ${ }^{12} \mathrm{CO}$ cloud ensembles, labeled \#2 and \#3, appear to extend in the direction of increasing Galactic longitude (see Figs. 4 and 5b). They correspond to the two main peaks of the latitude-velocity diagram integrated over the $l=27^{\circ}-29^{\circ}$ longitude range (see Fig. $3 \mathrm{~b}$ ) and the two line components observed in the $60-120 \mathrm{~km} \mathrm{~s}^{-1}$ range for both $\mathrm{H} \mathrm{I}$ and ${ }^{12} \mathrm{CO}$ (see Fig. 1). The comparison of Figs. 5a,b shows that they are mostly confined 


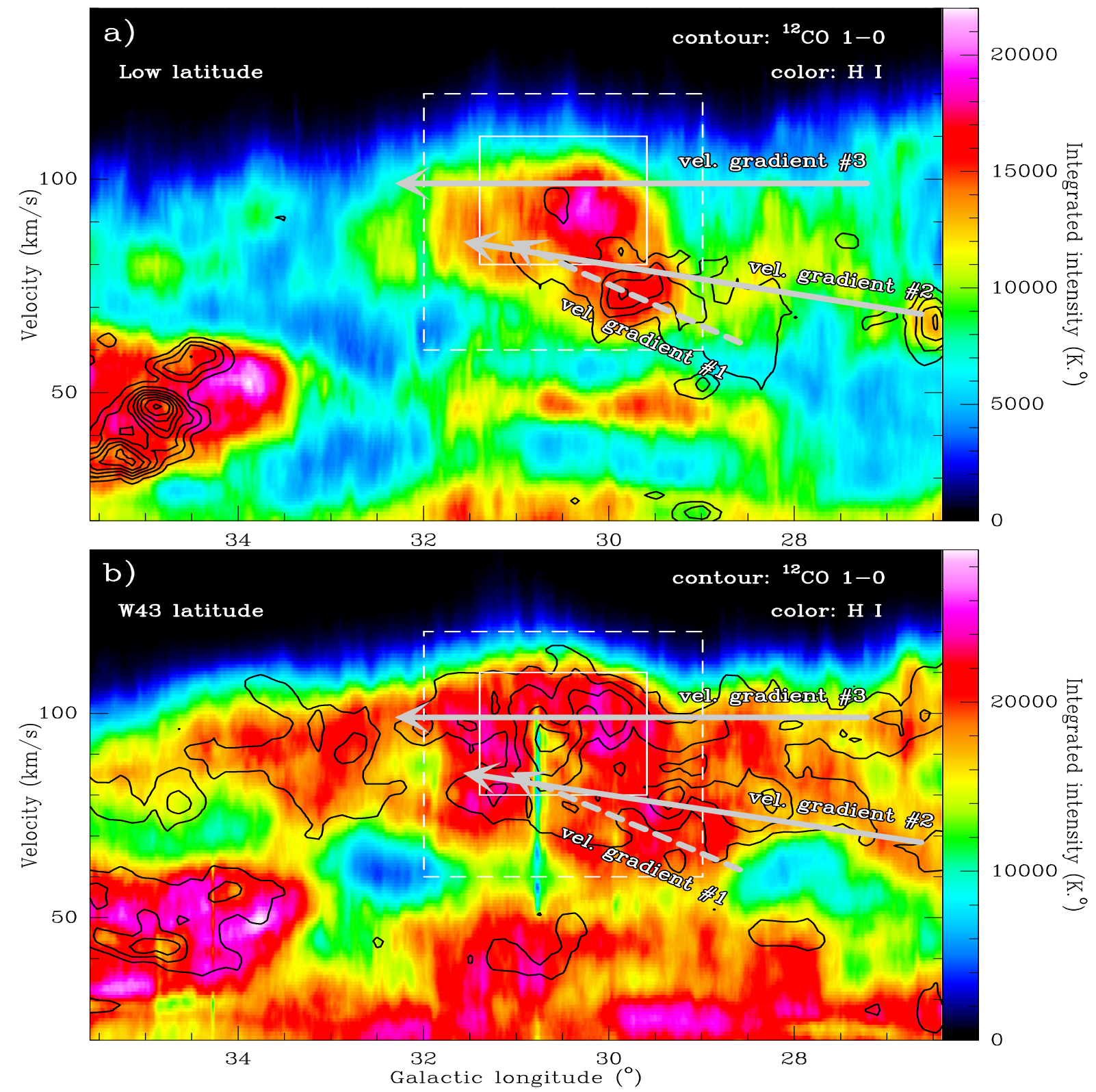

Fig. 5. Position-velocity diagrams investigating the ${ }^{12} \mathrm{CO} 1-0 / \mathrm{H} \mathrm{I}$ cloud ensembles \#1-\#3 identified in Figs. 3, 4 (white arrows, see also Sect. 3.3) at various latitudes. The distributions of the $\mathrm{HI}$ (color) and ${ }^{12} \mathrm{CO} 1-0$ (contours) are plotted against the Galactic longitude, kept as the $x$ axis, as in Fig. 2. The $\mathrm{HI}$ and ${ }^{12} \mathrm{CO} 1-0$ lines have been summed in a) over $b=-1.4^{\circ}$ to $b=-0.7^{\circ}$ to highlight negative-latitude cloud ensembles and in b) over the $b=-0.5^{\circ}$ to $b=0.3^{\circ}$ latitude range of the W43 molecular complex. The contours of a) go from 150 to $1200 \mathrm{~K}$ with a step of $150 \mathrm{~K}$. Those of b) run from 300 to $1200 \mathrm{~K}$, with a step of $300 \mathrm{~K}$. The white boxes represent the longitude extent and velocity range of the W43 molecular complex (continuous lines) and its $\mathrm{H}$ I envelope (dashed lines). The negative-latitude cloud ensemble \#1 can easily be identified in a) and the other two cloud ensembles can be seen in $\mathbf{b}$ ).

within the latitude range of the W43 molecular complex (from $b=-0.5^{\circ}$ to $b=0.3^{\circ}$ ), and inspection of Fig. 4 shows that they almost disappear in the $l=32^{\circ}-34^{\circ}$ longitude range. The velocity gradients of these ${ }^{12} \mathrm{CO}$ cloud ensembles are directly linked to the velocity structure of the W43 complex as seen from the ${ }^{12} \mathrm{CO}$ position-velocity diagram of Fig. $5 \mathrm{~b}$. This is even more obvious when inspecting the ${ }^{13} \mathrm{CO} 1-0$ longitude-velocity diagram of Nguyen Luong et al. (2011b) (see their Fig. 6) and the higher angular resolution ${ }^{13} \mathrm{CO} 2-1$ diagram of Carlhoff et al. (2013) (see their Fig. 5a). Both authors have proposed that the velocity component observed at $60-78 \mathrm{~km} \mathrm{~s}^{-1}$ is remotely associated with the W43 molecular complex, with a possible distance between them of a few hundred parsecs. Cloud ensemble \#2 begins at lower longitude and lower velocities and reaches the $\mathrm{W} 43$ position and velocity range with an approximate velocity gradient of $\sim 0.02 \mathrm{~km} \mathrm{~s}^{-1} \mathrm{pc}^{-1}$ over a distance of $\sim 450 \mathrm{pc}$ (see Figs. 4 and $5 \mathrm{~b}$ ). The ${ }^{12} \mathrm{CO}$ cloud ensemble \#3 is itself found at $\sim 97 \pm 7 \mathrm{~km} \mathrm{~s}^{-1}$, extending for a distance of $900 \mathrm{pc}$ (from $l \sim 25^{\circ}$ to $l \sim 34^{\circ}$ ), with several clouds peaking in the $\sim 92-100 \mathrm{~km} \mathrm{~s}^{-1}$ range (see Fig. 4). These two ${ }^{12} \mathrm{CO}$ cloud ensembles are associated with enhancements of the $\mathrm{HI}$ emission that are generally located at $\sim 5 \mathrm{~km} \mathrm{~s}^{-1}$ lower velocities than the ${ }^{12} \mathrm{CO}$ clouds (see Figs. 5a,b).

These three velocity gradients, indicated with arrows in Figs. 3-5, are associated with ensembles of clouds that are clear peaks in the position-velocity diagrams. They are not a direct proof of converging/colliding clouds since they could just underline stable clouds following the structure of the Milky Way 
F. Motte et al.: Atomic-to-molecular transition and putative colliding clouds in W43
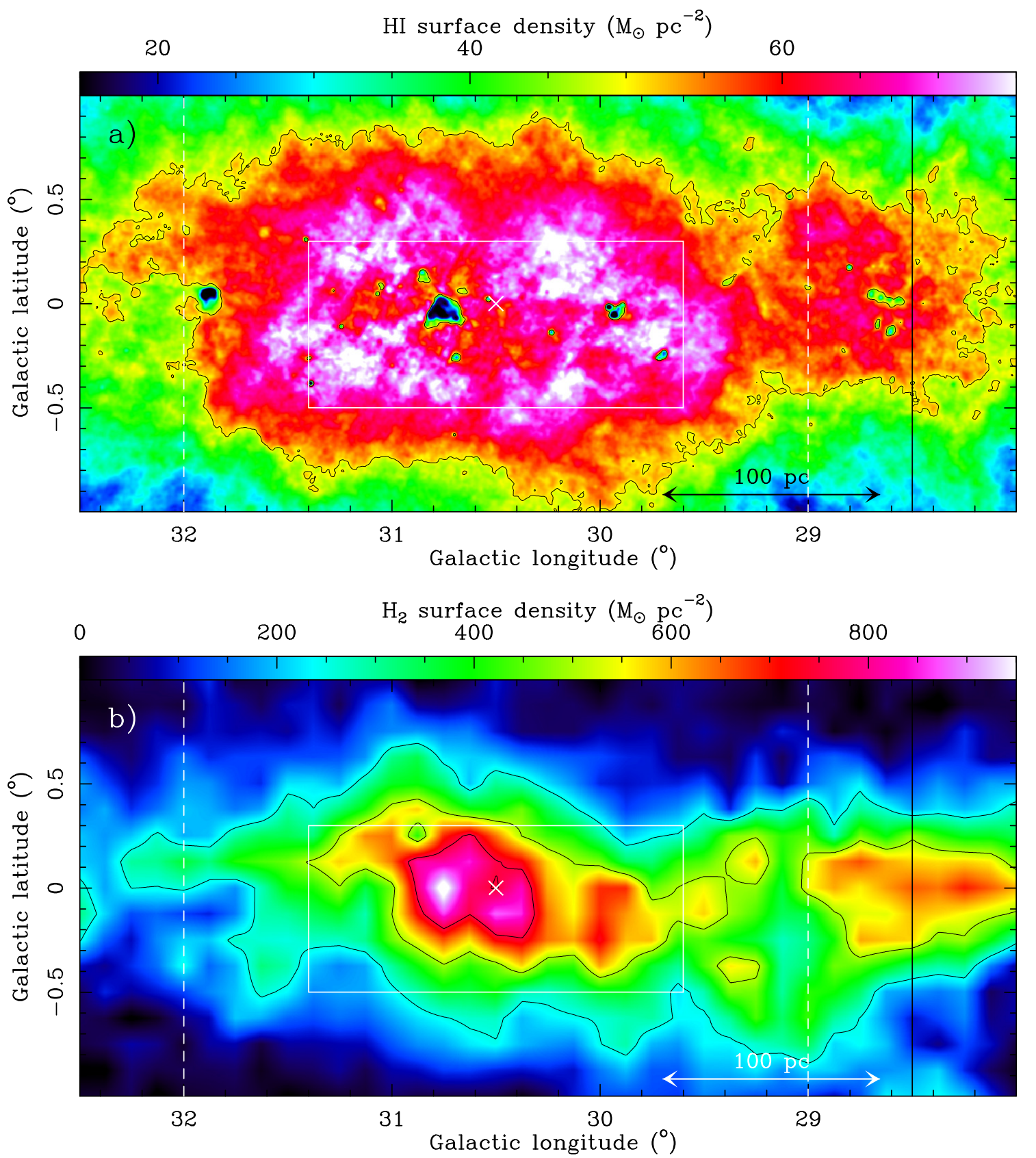

Fig. 6. W43 surface density images of $\mathrm{HI}$ and $\mathrm{H}_{2}$ gases in a) and b), respectively. They were calculated using $\mathrm{HI}$ and ${ }^{12} \mathrm{CO} 1-0$ lines integrated over $60-120 \mathrm{~km} \mathrm{~s}^{-1}$ and Eqs. (1) and (3). Contours are $50 M_{\odot} \mathrm{pc}^{-2}$ in a) and 250, 500, and $750 M_{\odot} \mathrm{pc}^{-2}$ in b). The white boxes outline the extent of the W43 molecular complex (continuous lines) and its H I envelope (dashed lines) as defined in Nguyen Luong et al. (2011b). The black box locates the largest ring used in the $R_{\mathrm{H}_{2}}$ diagram (see Fig. 7) and the white cross its center.

in the region surrounding W43. Nevertheless, it is tempting to check further that they could be the remnants of H I gas streams and/or ${ }^{12} \mathrm{CO}$ streaming clouds that could have collided to form the W43 molecular complex.

\section{Discussion: Tracing back the formation of the W43 molecular complex}

$\mathrm{H}_{2}$ molecules and $\mathrm{H} \mathrm{I}$ atoms are directly linked by formation and destruction processes. In the diffuse parts of cloud complexes, $\mathrm{H}$ I gas forms from the dissociation of $\mathrm{H}_{2}$ molecules by the general interstellar radiation field of the Galactic plane, by the local UV radiation arising from embedded OB clusters, or by cosmic rays. In contrast, the high-density regions of cloud complexes offer enough shielding from HI atoms (cold and hot WNM), $\mathrm{H}_{2}$ molecules, and dust so that $\mathrm{HI}$ atoms efficiently turn into $\mathrm{H}_{2}$ molecules on the dust grains surfaces. Observing the formation of $\mathrm{H}_{2}$ gas and molecular clouds from $\mathrm{HI}$ in an envelope or streams is therefore a challenge.

\subsection{A surface density threshold for the atomic gas?}

Hypothetically, if there is equilibrium between the formation and destruction of $\mathrm{H}_{2}$, there should be a steep transition from atomic to molecular gas associated with the saturation of the H I surface density. A value of $\Sigma_{\mathrm{H}_{\mathrm{I}}} \sim 10 M_{\odot} \mathrm{pc}^{-2}$ has been observed in extragalactic studies (see, e.g., Blitz \& Rosolowsky 2006; Bigiel et al. 2008; Wong et al. 2009). Similar saturation levels 
have recently been found for diffuse and dense Galactic clouds (e.g., Barriault et al. 2010; Lee et al. 2012): $\Sigma_{\mathrm{H} \text { I }} \sim 4-7 M_{\odot} \mathrm{pc}^{-2}$, equivalent to $N_{\mathrm{H} \text { I }} \sim 5-8 \times 10^{20} \mathrm{~cm}^{-2}$. The equilibrium model of Krumholz et al. (2009) does predict such a surface density threshold at $\Sigma_{\mathrm{H}}=10 M_{\odot} \mathrm{pc}^{-2}$ for solar metallicity clouds. In contrast, the dynamical models accrete streams/clouds of $\mathrm{HI}$ and $\mathrm{H}_{2}$ gases to form molecular clouds/complexes that are in neither kinematic nor chemical equilibrium. As a consequence, dynamically formed molecular clouds should display several transitions from atomic to molecular media along each line-of-sight. The model of, say, Glover et al. (2010) predicts that the H I surface density is high when the cloud is assembling, that it then drops sharply within 1-2 Myr, but that it may never reach equilibrium by 20-30 Myr.

To measure the surface density map of atomic gas in W43 (see Fig. 6b), we used the H I VGPS data integrated over the $60-120 \mathrm{~km} \mathrm{~s}^{-1}$ range as in Fig. 2 and Sect. 3.3. We assumed that the H I emission was mainly optically thin and took a distance of $5.5 \mathrm{kpc}$ for W43 and its surroundings. The equation of Spitzer (1978) for the column density turns into the following equation for the $\mathrm{HI}$ surface density, $\Sigma_{\mathrm{HI}}$, as a function of the $\mathrm{H}$ I line brightness, $T_{\mathrm{MB}}(\mathrm{HI})$ :

$\Sigma_{\mathrm{HI}} \simeq 0.014 M_{\odot} \mathrm{pc}^{-2} \times \frac{\int_{60}^{120} T_{\mathrm{MB}}(\mathrm{HI}) \mathrm{d} v}{1 \mathrm{~K} \mathrm{~km} \mathrm{~s}^{-1}}$.

Figure 7 assumes elliptical geometry (see Sect. 3.2) and displays the mean H I surface density across the W43 atomic/molecular complex and its surroundings. We computed $\Sigma_{\mathrm{HI}}$ in rectangular annuli centered at $l=30.5^{\circ}$ and $b=0^{\circ}$, with an aspect ratio $3: 2$, separated by $\sim 15 \mathrm{pc}$ and $\sim 10 \mathrm{pc}$ along the Galactic longitude and latitude, and reaching up to regions covering the cloud ensembles identified in Sect. 3.3. In regions where the H I lines are clearly optically thick, the surface density of $\mathrm{HI}$ is underestimated. Outside these areas, i.e. at the $60-170 \mathrm{pc}$ radii of Fig. $7, \Sigma_{\mathrm{HI}}$ steadily decreases with radius from $\sim 82 M_{\odot} \mathrm{pc}^{-2}$ to $\sim 36 M_{\odot} \mathrm{pc}^{-2}$. While the gradient of the atomic gas surface density is robust, its absolute value is uncertain by at least a factor of $\sim 2$. The main reason is that we have estimated $\Sigma_{\mathrm{HI}}$ by integrating the $\mathrm{HI}$ lines over the ${ }^{13} \mathrm{CO}$ velocity range, which may not apply perfectly for H I (see Sect. 3.1). Since the line widths of $\mathrm{HI}$ and $\mathrm{CO}$ gases in clouds are generally found to be similar (e.g., Barriault et al. 2010), a factor of $\sim 2$ uncertainty seems reasonable. Where the emission is optically thick, this introduces additional uncertainty, especially in the inner parts of W43, at radii smaller than $60 \mathrm{pc}$.

The surface density of the HI gas in W43 is in clear disagreement with previous saturation findings in nearby Galactic clouds (compare the red curve of Fig. 7 to, e.g., Lee et al. 2012) and resolved galaxies (Bigiel et al. 2008), as well as with the threshold predicted by Krumholz et al. (2009). Indeed, $\Sigma_{\mathrm{HI}}$ is about 6.5 times greater than $10 M_{\odot} \mathrm{pc}^{-2}$ and displays a negative radial gradient. The modeled $\mathrm{H}_{\mathrm{I}}$ threshold $\Sigma_{\mathrm{HI}}=10 \mathrm{M}_{\odot} \mathrm{pc}^{-2}$ is strictly valid for the idealized case of a spherical molecular cloud with solar metallicity and where $\mathrm{HI}$ and $\mathrm{H}_{2}$ gases are mutually exclusive. Krumholz et al. (2009) extrapolate it to the case of more structured molecular entities, such as molecular complexes composed of several clouds that host OB clusters. They show that $\Sigma_{\mathrm{HI}}$ is, to a good approximation, independent of the strength of the interstellar radiation field and sublinearly dependent on the metallicity. The W43 molecular complex, with a slightly higher metallicity (possibly $1.4 \times Z_{\odot}$, Nguyen Luong et al. 2011b) and a very strong mean interstellar radiation field $\left(20 \times G_{0}\right.$ according to Shibai et al. 1991 and

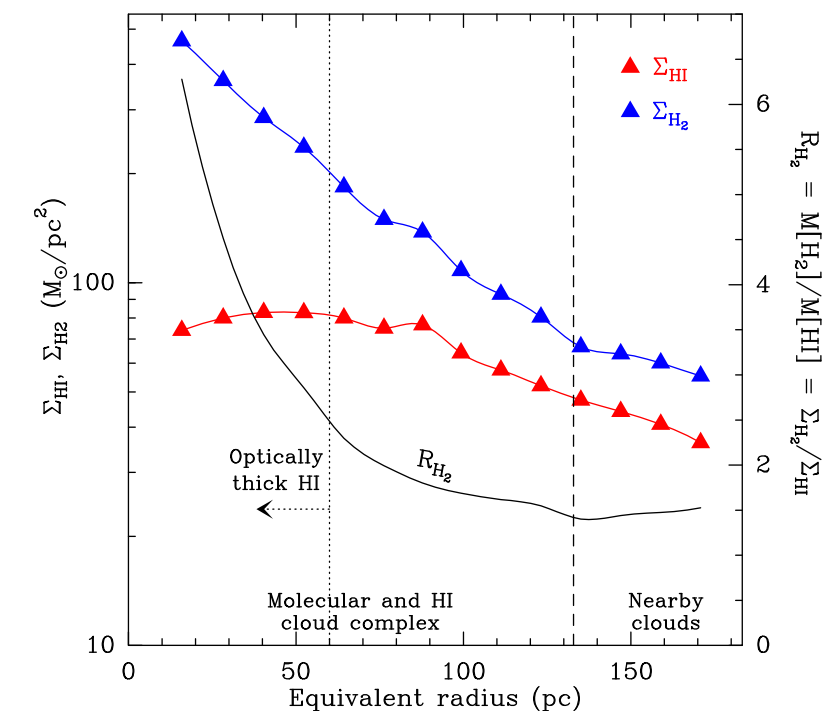

Fig. 7. $\mathrm{HI}$ and $\mathrm{H}_{2}$ surface densities (red and blue curves plus triangles, respectively) and the $\mathrm{H}_{2}$-to-H I ratio (black curve) measured throughout the W43 atomic/molecular cloud complex and the nearby clouds. Measurements are averaged within rectangular annuli centered at $l=30.5^{\circ}$ and $b=0^{\circ}$ and with an aspect ratio 3:2. The dashed vertical line outlines the extent of the W43 complex, the dotted vertical line and arrow locate the region inside which the $\mathrm{HI}$ line emission is widely optically thick. The HI surface density is significantly higher than the equilibrium models predict, while the $\mathrm{H}_{2}$ density remains high, in disagreement with a clear $\mathrm{HI} / \mathrm{H}_{2}$ transition.

possibly $100-1000 \times G_{0}$, Schneider, priv. com.), is therefore predicted to display an $\mathrm{HI}$ threshold around $10 M_{\odot} \mathrm{pc}^{-2}$. When focusing on a single $10 \mathrm{pc}$ cloud such as W43-Main, the measured H I surface density decreases by a factor of a few but is still a factor of $\sim 3$ times higher than $10 M_{\odot} \mathrm{pc}^{-2}$. The high H I surface densities observed here are a consequence of either numerous $\mathrm{H}_{2}$ to $\mathrm{HI}$ transitions along each line of sight or a non-steady state formation such as suggested by the Glover \& Mac Low (2007) models. In the case of $\mathrm{W} 43$, both processes probably need to be taken into account. We recall that the W43 molecular cloud complex remains a coherent and gravitationally bound cloud structure, even if it is structured as several individual clouds and numerous layers of $\mathrm{H}_{2}$ and $\mathrm{H} \mathrm{I}$ gas.

\subsection{The transition from atomic to molecular gas}

The transition from atomic to molecular gas can also be traced by the evolution of the $\mathrm{H}_{2}$-to-H I ratio from the diffuse $\mathrm{H}$ I envelope to the dense molecular cloud. We estimated the mean $\mathrm{H}_{2}$-toH I ratio, $R_{\mathrm{H}_{2}}$, throughout $\mathrm{W} 43$ and its surroundings by computing the mass surface density ratio of molecular $\mathrm{H}_{2}$ and atomic $\mathrm{H} \mathrm{I}$ gas. The H I surface density map shown in Fig. 6a and computed from Eq. (1) has been smoothed to the 450" (or $12 \mathrm{pc}$ ) resolution of the CfA data. Associated uncertainties are discussed in Sect. 4.1.

We determined the $\mathrm{H}_{2}$ mass surface density from the total intensity of ${ }^{12} \mathrm{CO} 1-0 \mathrm{CfA}$ data, $T_{\mathrm{MB}}\left({ }^{12} \mathrm{CO}\right)$, integrated again over $60-120 \mathrm{~km} \mathrm{~s}^{-1}$. Adopting a value for the CO-to$\mathrm{H}_{2}$ conversion factor (also known as the $X$ factor) given by Bloemen et al. (1986), the molecular column density, $N_{\mathrm{H}_{2}}$, is estimated through

$N_{\mathrm{H}_{2}}=2.75 \times 10^{20} \mathrm{~cm}^{-2} \times \frac{\int_{60}^{120} T_{\mathrm{MB}}\left({ }^{12} \mathrm{CO}\right) \mathrm{d} v}{1 \mathrm{Km} \mathrm{s}^{-1}}$. 
With a mean molecular weight of $\mu=2$, the mass surface density of molecular gas, $\Sigma_{\mathrm{H}_{2}}$, can be computed by the following equation:

$\Sigma_{\mathrm{H}_{2}} \simeq 4.41 M_{\odot} \mathrm{pc}^{-2} \times \frac{\int_{60}^{120} T_{\mathrm{MB}}\left({ }^{12} \mathrm{CO}\right) \mathrm{d} v}{1 \mathrm{~K} \mathrm{~km} \mathrm{~s}^{-1}}$.

Figure $6 \mathrm{~b}$ gives the surface density map of molecular gas traced by ${ }^{12} \mathrm{CO} 1-0$. The $\mathrm{H}_{2}$ surface density values are uncertain by at least a factor of two because the $X$ factor, converting $\mathrm{CO}$ emission into $\mathrm{H}_{2}$ mass, is expected to vary across the Milky Way. According to Shetty et al. (2011), the $X$ factor only makes sense as a cloud-scale average. However, the relations observed in Figs. 7, 8 should be secure since cloud properties are investigated through ${ }^{12} \mathrm{CO}$ emission averaged over one to one hundred beams of $H P B W \simeq 13 \mathrm{pc}$. Moreover, given that W43 lies in the inner part of the Galactic disk, the amount of $\mathrm{CO}$-dark $\mathrm{H}_{2}$ gas should be minimal. Instead of the surface density ratios used by e.g. Lee et al. (2012), we could have computed mass ratios, since they are strictly equal:

$R_{\mathrm{H}_{2}}=f_{\mathrm{H}_{2}} / f_{\mathrm{HI}_{\mathrm{I}}}=M_{\mathrm{H}_{2}} / M_{\mathrm{HI}}=\Sigma_{\mathrm{H}_{2}} / \Sigma_{\mathrm{HI}}$,

where the $\mathrm{H}_{2}$ and $\mathrm{HI}$ gas fractions are defined by $f_{\mathrm{H}_{2}}=$ $M_{\mathrm{H}_{2}} /\left(M_{\mathrm{HI}}+M_{\mathrm{H}_{2}}\right)$ and $f_{\mathrm{HI}}=M_{\mathrm{HI}} /\left(M_{\mathrm{HI}_{\mathrm{I}}}+M_{\mathrm{H}_{2}}\right)$.

In Fig. 7, we see that the $\mathrm{H}_{2}$-to-H I ratio rises smoothly with decreasing radii with values of $R_{\mathrm{H}_{2}} \sim 1.5$ at the outskirts of the complex (i.e., at a radius of $135 \mathrm{pc}$ ) and $\sim 6$ at $16 \mathrm{pc}$ from the center of the complex. Even if we exclude the regions inside which the $\mathrm{HI}$ lines are clearly optically thick, and therefore focusing on the outer parts of what we called the H I envelope in Sect. 3.2, we see that the $R_{\mathrm{H}_{2}}$ ratio still increases by about a factor of two as we move toward the center of the complex. Figure 7 therefore suggests that $\mathrm{H}$ I turns into $\mathrm{H}_{2}$ more efficiently at greater depths within the W43 complex. The surroundings of the W43 complex (called nearby clouds in Fig. 7) display a slight increase in $R_{\mathrm{H}_{2}}$ from the $\sim 1.5$ value measured at the outskirts of the complex. This behavior marks the start of new molecular cloud structures, confirming our definition of the W43 atomic/molecular complex.

The transition from $\mathrm{HI}$-dominated to $\mathrm{H}_{2}$-dominated gas is usually set at either $R_{\mathrm{H}_{2}}=0.25$ or $R_{\mathrm{H}_{2}}=0.1$ (see, e.g., Lee et al. 2012, and references therein). With either definition, the observed molecular ratio (Fig. 7) surprisingly suggests that this transition happens outside the H I envelope that we have identified for W43. As previously mentioned, the absolute values of $\Sigma_{\mathrm{HI}}\left(\right.$ or $M_{\mathrm{HI}}$ ) and $\Sigma_{\mathrm{H}_{2}}\left(\right.$ or $M_{\mathrm{H}_{2}}$ ) are both uncertain by factors of at least two. Even when accounting for these uncertainties (i.e., corresponding to values of $R_{\mathrm{H}_{2}}$ correct to within a factor 4), we cannot reconcile the molecular ratio of Fig. 7 and our picture of a molecular complex surrounded by an $\mathrm{H}$ I envelope (see Sect. 3.2). Extending Fig. 7 to larger radii would not make sense since wider annuli would cover gas from other molecular structures observed at greater distances (see Fig. 2 and Sect. 4.3).

If we take the $\Sigma_{\mathrm{HI}}$ and $R_{\mathrm{H}_{2}}$ curves of Fig. 7 at face value, the $\mathrm{W} 43$ complex seems to develop/form in an area overall shielded against the Galactic radiation field. This is in direct agreement with the high global molecular mass fraction of W43 (Nguyen Luong et al. $2011 \mathrm{~b}, \sim 65 \%$, ) which follows the general decrease with the Galactic radius, observed for other molecular clouds (Elmegreen \& Elmegreen 1987). Figure 8 plots the value of $R_{\mathrm{H}_{2}}$ for each map point within the rectangle outlined in Fig. 2 and therefore containing both the W43 complex and nearby clouds. As a function of their total surface density $\left(\Sigma_{\mathrm{H}_{\mathrm{I}}}+\Sigma_{\mathrm{H}_{2}}\right)$, the distribution of points cannot be fit by the fiducial model of Krumholz et al. (2009) with a solar metallicity, and instead requires a

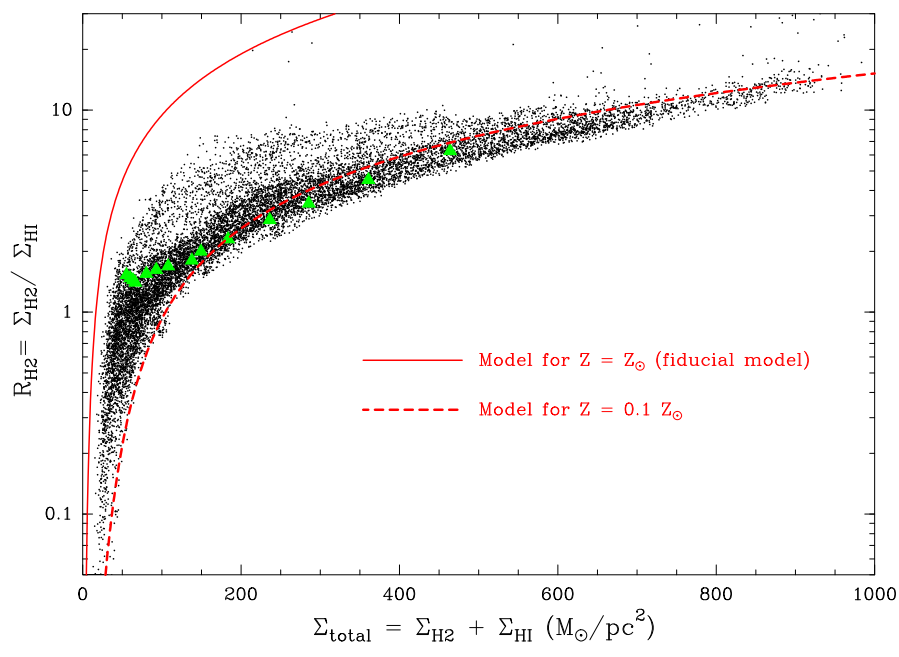

Fig. 8. $\mathrm{H}_{2}$-to-H I ratio, $R_{\mathrm{H}_{2}}$, of each map point as a function of the total surface density, $\Sigma_{\mathrm{H}_{\mathrm{I}}}+\Sigma_{\mathrm{H}_{2}}$, compared with the predictions of the Krumholz et al. (2009) model (red curves, taken from their Eq. (39)). The green triangles indicate the mean $R_{\mathrm{H}_{2}}$ values measured within the rings used in Fig. 7. The $R_{\mathrm{H}_{2}}$ ratio values measured throughout the W43 molecular complex and its surroundings are in striking disagreement with the fiducial model of Krumholz et al. (2009).

much lower and unrealistic metallicity: $0.1 \times Z_{\odot}$ rather than the value of $1.4 \times Z_{\odot}$ found by Nguyen Luong et al. (2011b). It also confirms that the W43 region, including the H I envelope, lies in a domain already shielded from external sources of UV radiation. Indeed, Fig. 8 does not trace the turnover between atomic-dominated and molecular-dominated zones observed by Lee et al. (2012, see their Fig. 12) and predicted by Krumholz et al. (2009). The purely atomic envelope of W43 can be seen at high latitude but does not seem to exist within the Galactic disk. This can be explained by the high gas and stellar pressure that should exist in the W43 region. Indeed, Blitz \& Rosolowsky (2006) show that, in galaxies, there is a relation between the combined pressures from stars and gas and the molecular ratio of clouds. Gas structures associated with the velocity gradients found in Sect. 3.3 are molecular and have a complex structure well before any interaction with W43, which argues for their being called cloud ensembles rather than gas streams.

From Fig. 7, we also see that $\Sigma_{\mathrm{H}_{\mathrm{I}}}$ and $R_{\mathrm{H}_{2}}$ simultaneously increase within the outer part of the $\mathrm{HI}$ envelope as the radius decreases from $135 \mathrm{pc}$ to $60 \mathrm{pc}$. This increase in the H I surface density suggests a continuous accumulation of $\mathrm{HI}$ gas in this heterogeneous $\mathrm{H}_{2} / \mathrm{H}$ I structure, even if it is overall shielded from the Galactic field and protected from the UV field of the embedded $\mathrm{H}$ II regions. In this scenario the $\mathrm{HI}$ and $\mathrm{H}_{2}$ gases are well-mixed, coexisting but far from chemical equilibrium, as proposed by the models of Heitsch \& Hartmann (2008) or Clark et al. (2012), among others. That some $\mathrm{H}_{2}$ is likely being converted back into $\mathrm{HI}$ is demonstrated by the strong $\left(3 \times 10^{5} L_{\odot}\right)$ and large-scale $\left(1^{\circ} \times 1^{\circ}\right) \mathrm{C}$ II emission extended over the complete W43 complex, which is most probably associated with its many photodissociation layers (Shibai et al. 1991). The increase in the $\mathrm{H}_{2}$-to-H I ratio itself traces the formation of molecular clouds thanks to $\mathrm{H}_{2}$ molecules forming and the molecular medium becoming denser. This also happens far from the area already defined as the W43 molecular cloud complex. If confirmed, these two results point toward a dynamical and nonsteady state scenario for the formation of the W43 molecular complex. 


\subsection{Clouds following the Galactic structure/kinematics}

In the immediate surroundings of the W43 molecular complex, we have identified three cloud ensembles associated with structured ${ }^{12} \mathrm{CO}$ and H I emission (see Fig. 4 and Sect. 3.3). With a common distance of $5.5 \mathrm{kpc}$, these three ensemble of clouds are developing $\sim 300 \mathrm{pc}$ away from W43, while their spatial and velocity distributions suggest that they join at the location of the W43 molecular complex (see arrows in Figs. 3-5). To position these clouds in the W43 region, one should remember that W43 is located close to the tangent point of the Scutum-Centaurus arm and probably at or close to its connecting point with the long bar of the Milky Way.

Cloud ensemble \#1 originates in longitudes smaller than that of W43 and approaches W43 from below the Galactic plane (see Figs. 3b, 4, and 5a). It could well be a high-latitude cloud attracted by the gravitational potential well of the Galactic plane and W43.

The two main cloud ensembles (\#2 and \#3) are themselves confined at low latitude and are seen at longitudes smaller than that of W43: from $b=-0.5^{\circ}$ to $b=0.3^{\circ}$ and from $l=27^{\circ}$ to $l=30^{\circ}$ (see Figs. 3,4 ). Cloud ensemble \#2 follows a velocity gradient close to the one found for the leading part of the Scutum-Centaurus arm, shown schematically in Fig. 4 (e.g., Vallée 2008; Dame \& Thaddeus 2011). Cloud ensemble \#3 has a velocity gradient consistent with the preceding part of the Scutum-Centaurus arm as defined by Vallée (2008, see Fig. 4). However, the new model of the Milky Way by, e.g. Dame \& Thaddeus (2011) stops the spiral structure of the ScutumCentaurus arm at the location of W43 and starts the long bar a few degrees away (López-Corredoira et al. 2007; Benjamin et al. 2005; Rodríguez-Fernández \& Combes 2008). Therefore, cloud ensemble \#3 may alternatively correspond to clouds at the tip of the long bar (see also González-Fernández et al. 2012). As shown in Fig. 4, the velocity gradients of cloud ensembles \#2 and \#3 do not lie on the median location of the ScutumCentaurus arm modeled with circular orbits (compare the arrows and the thick gray curve). We indeed measured $\sim 1^{\circ}$ and up to $\sim 20 \mathrm{~km} \mathrm{~s}^{-1}$ offsets between the observed cloud ensembles and the position-velocity structure predicted by Vallée (2008).

These offsets for a single cloud could easily be explained by streaming motions of gas in and out of the gravitational well of spiral arms. However, in the case of clouds spanning $\sim 500 \mathrm{pc}$ along the Galactic plane through and around W43, we favor an explanation related to the interaction with the long bar. Cloud ensembles \#2 and \#3 could therefore simply follow the structure of the Milky Way without interacting much with each other or with W43. Nevertheless, their location near the tip of the Galactic bar makes them subject to both the spiral arm circular rotation and the solid-body bar elliptical rotation. According to numerical simulations, such locations in galaxies have a high probability of cloud-cloud collision due to orbit crowding (e.g., Rodríguez-Fernández \& Combes 2008).

\subsection{Clouds streaming toward W43?}

With the refined $5.5 \mathrm{kpc}$ distance and the velocity range discussed in Sect. 3.1, we recomputed the mass of the W43 complex, whose Galactic limits have been set by Nguyen Luong et al. (2011b) (see Fig. 2, area of 130 pc equivalent diameter). Without any correction for optical thickness, the atomic $\mathrm{H} \mathrm{I}$ and molecular $\mathrm{H}_{2}+\mathrm{He}$ gas masses of W43 are $M_{\mathrm{HI}} \sim 9 \times 10^{5} M_{\odot}$ and $M_{\mathrm{H}_{2}+\mathrm{He}} \sim 8 \times 10^{6} M_{\odot}$, leading to a total gas mass of $M_{\mathrm{W} 43} \sim 9 \times 10^{6} M_{\odot}$. With the same assumptions, the mass of the H I envelope surrounding W43 (see Fig. 2, area of $270 \mathrm{pc}$ equivalent diameter) is $M_{\mathrm{H} \text { I, env }} \sim 3 \times 10^{6} M_{\odot}$. With $\sim 10^{7} M_{\odot}$ of gas, the $\mathrm{W} 43{ }^{12} \mathrm{CO} / \mathrm{H}$ I complex is a large gravitational potential well that may attract nearby molecular clouds of $\sim 10^{5} M_{\odot}$ mass. Here we briefly investigate the kinematic state of the three cloud ensembles identified in Sect. 3.3, with the aim of evaluating whether they are streaming toward W43.

That these three cloud ensembles meet at the location of W43 could reflect a real physical interaction. The first argument is that the velocity dispersion measured for $\mathrm{W} 43$ is large $\left(F W H M^{13} \mathrm{CO} \sim\right.$ $22.3 \mathrm{~km} \mathrm{~s}^{-1}$; see Nguyen Luong et al. 2011b) and could better account for agglomeration of clouds than micro-turbulence. This phenomenon is called gravitationally driven turbulence in dynamical models of cloud formation (see, e.g., Heitsch et al. 2008). Cloud ensembles \#1 to \#3 could thus be streams that are accreted onto the central W43 region, driven simply by gravity. This accretion from the ambient surrounding gas and at a late stage in the formation of molecular cloud complexes has been noted in the numerical models of Vázquez-Semadeni et al. (2007), Heitsch \& Hartmann (2008), and Dobbs (2008), among others.

Velocity gradients of molecular clouds are often interpreted as tracing their specific angular momentum, which can be compared with Galactic rotation models and predictions of cloud formation scenarios (e.g., Imara \& Blitz 2011). We do not investigate this idea here since cloud ensembles \#1 to \#3 are defined on much larger scales than that of individual clouds, and ensembles \#2 and \#3 tend to reflect the velocity structure of the Milky Way (see Sect. 4.3). This last point implies that the major part of the gradients observed in the direction of increasing longitude does not trace the infall velocities of the cloud ensembles onto W43. Since cloud ensemble \#1 is mainly observed in the direction of decreasing latitude, its velocity gradient could be compared more meaningfully to its predicted free-fall velocity toward the $\mathrm{W} 43{ }^{12} \mathrm{CO}$ complex. In fact, the $\sim 0.2 \mathrm{~km} \mathrm{~s}^{-1} \mathrm{pc}^{-1}$ gradient over $\sim 40 \mathrm{pc}$ of Galactic latitude is consistent with the freefall velocity of clouds located $\sim 200 \mathrm{pc}$ away from the $\sim 10^{7} \mathrm{M}_{\odot}$ W43 complex. Interestingly, the velocity dispersion of cloud ensemble \#1, which is estimated through ${ }^{12} \mathrm{CO}$ line widths, narrows when approaching W43, with the FWHM decreasing from $\sim 20 \mathrm{~km} \mathrm{~s}^{-1}$ to $<10 \mathrm{~km} \mathrm{~s}^{-1}$ (see Fig. 5a), again suggesting that it is attracted by the deep gravitational potential well of W43. We do not see any acceleration of the infall with decreasing distance of the cloud ensemble \#1 to W43, but this can be covered up easily by projection and rotation effects, as well as by cloud inhomogeneities. As for cloud ensembles \#2 and \#3, they are initially separated by $>40 \mathrm{~km} \mathrm{~s}^{-1}$ velocities along the line of sight and extend within the W43 complex as velocity structures. The complex structure in velocity of W43 is also confirmed by its velocity dispersion, which is much larger than that of individual cloud ensembles streaming toward it: $F W H M_{12}^{\mathrm{W} 43} \sim 30 \mathrm{~km} \mathrm{~s}^{-1}$ for W43 vs. $\sim 10 \mathrm{~km} \mathrm{~s}^{-1}$ for nearby clouds (see Fig. 4). The $\sim 20 \mathrm{~km} \mathrm{~s}^{-1}$ velocity jumps observed in the densest parts of the W43 complex, such as the W43-Main and W43-South clouds (see Fig. 4), could represent local and abrupt accelerations of the infall.

According to Carlhoff et al. (2013), the W43 ${ }^{13} \mathrm{CO}$ molecular complex is stable against Galactic shear. The ${ }^{12} \mathrm{CO} / \mathrm{H}$ I cloud complex, which extends to much greater distances (from the white to the white-dashed rectangle in Fig. 2) and the $100 \mathrm{pc}$ ensemble of clouds located at its outskirts (from the whitedashed to the black rectangle in Fig. 2) should be far more sensitive to Galactic shear. Indeed, despite their huge total mass, 
up to $\sim 10^{7} M_{\odot}$, the large area they cover, $\sim 6-8 \times 10^{4} \mathrm{pc}^{2}$, and their velocity range up to $60 \mathrm{~km} \mathrm{~s}^{-1}$ lead to a shear parameter of $S_{\mathrm{g}}=1-3$ (with Eq. (8) of Dib et al. 2012). In a simple spiral rotation model of the Milky Way, both the envelope and nearby clouds would thus not be linked to W43. However, numerical simulations show that areas in front of galactic bars are locations where clouds sporadically agglomerate (e.g., Athanassoula 1992; Wozniak 2007; Renaud et al. 2013). Other indirect arguments come from extragalactic observational studies that found extreme molecular cloud complexes at the ends of galactic bars (e.g., M 83, NGC 1300; see Martin \& Friedli 1997, and references therein).

To conclude, we do not find definitive proof that nearby clouds are streaming toward W43, though, as discussed here, numerous factors favor this interpretation. W43, at a special location in the Milky Way, is denser and more dynamic than typical molecular clouds of the Galactic disk. These unusual characteristics have allowed us to identify clouds possibly streaming toward W43. Deeper observations and careful analyses of other cloud complexes may teach us whether cloud agglomeration occurs as frequently as it appears to within numerical simulations (see, e.g., Dobbs 2008).

\subsection{History of cloud and star formation in the region of W43}

Here we try to extend the interpretation that cloud ensembles are streaming toward W43 back to times when the W43 molecular cloud complex was just starting to form. According to numerical models, the special location of W43 in the Milky Way should have promoted its ability to accumulate $\mathrm{H}$ I and $\mathrm{H}_{2}$ gas from different structures (e.g., Rodríguez-Fernández \& Combes 2008). We have shown in Sect. 4.2 that most of the $\mathrm{H}_{2}$ molecules form far out of the classical $\sim 10-50 \mathrm{pc}$ outskirts of molecular clouds (e.g., Lee et al. 2012) and farther even than the 60-135 pc radii (from the center of the complex) taken to be the optically thin part of the H I envelope (see Fig. 7). The W43 molecular cloud complex may therefore have formed through the agglomeration of clouds containing both molecular and atomic gas. The clouds that currently are potentially streaming toward W43 (see Sect. 4.4) display velocity gradients following the Galactic structure. They thus can reasonably be taken as models for the initial cloud flows that created W43, but their surface density could be orders of magnitude higher or lower.

The level of cloud structure and the star formation activity of W43 and its nearby clouds can help define the chronology of the cloud and star formation events in the region. The molecular cloud structure \#2 qualifies as "pre-star forming" according to Sawada et al. (2012), who labeled it Cloud M-2. They argue that it is not yet structured at high density, with a moderate brightness distribution index, and that it hosts very few H II regions (Anderson \& Bania 2009). For comparison, W43 (their cloud H-2) is categorized as an "active star-forming complex" since it has a high brightness distribution index and contains many more $\mathrm{H}$ II regions.

Molecular cloud ensemble \#3 was not discussed by Sawada et al. (2012). It hosts many H II regions (Anderson \& Bania 2009) and an enhanced number of red super giant (RSG) clusters/associations (see, e.g., Fig. 9 of González-Fernández et al. 2012). The three main RSG associations of the first quadrant are indeed located around G29.2-0.2 (RSGC3 + Alicante7 + Alicante10, e.g. González-Fernández \& Negueruela 2012), G26.2-0.1 (RSGC2, also called Ste2, see, e.g., Negueruela et al. 2012), and G24.6+0.4 (RSGC1 + Alicante8, Negueruela et al. 2010). They contain numerous (30-50) RSG stars, are thus massive, $\sim 2-10 \times 10^{4} \quad M_{\odot}$ stellar associations. González-Fernández et al. (2012) locate them at the tip of the long bar of the Milky Way and $\sim 6 \mathrm{kpc}$ from the Sun. Their presence at $l=24^{\circ}-30^{\circ}$ suggests that, $\sim 20 \mathrm{Myr}$ ago, there were several sites of extremely active cloud and star formation located $\sim 500 \mathrm{pc}$ downstream of W43 (see Fig. 2). This spatial offset is reminiscent of those found between clouds and star clusters in the NGC 6951 galaxy (van der Laan et al. 2011), for instance. Molecular cloud ensemble \#3 is associated with these RSGCs and is one order of magnitude less massive than W43 (see Fig. 2). We thus propose that cloud agglomeration and star formation have started in the cloud complex that was associated with velocity gradient \#3. It is now enhanced in W43 mainly owing to clouds infalling from cloud ensemble \#2. This chronology agrees with numerical simulations of galaxy evolution with a barred potential, which tend to accumulate clouds in spiral leading arms ahead of the bar, form superstar clusters within these cloud complexes and then drag these clusters and clouds within the bar, where they often disrupt (Wozniak 2007; Renaud et al. 2013, and in prep.).

\section{Summary and conclusions}

To improve our knowledge of molecular cloud formation, we have initiated an investigation into W43, an extreme molecular cloud and star-forming complex located at a dynamical place within the Milky Way, $5.5 \mathrm{kpc}$ from the Sun. We used an H I and ${ }^{12} \mathrm{CO} 1-0$ database that allows us to trace back the formation of molecular clouds on $10 \mathrm{pc}$ to $400 \mathrm{pc}$ scales for this region. Our main findings can be summarized as follows:

1. We detected an HI envelope around the W43 molecular cloud (see Fig. 2), confirming the work of Nguyen Luong et al. (2011b). It has an aspect ratio of 3:2 (with the larger axis along the Galactic longitude), an equivalent diameter of $\sim 270 \mathrm{pc}$, and a mass of $\sim 3 \times 10^{6} M_{\odot}$. Interestingly, such a well-defined and symmetrical envelope is rarely seen around star-forming regions.

2. Three cloud ensembles of $\mathrm{HI}$ and ${ }^{12} \mathrm{CO}$ gas develop along hundreds of parsecs on the outskirts of and within the atomic envelope (see Figs. 3-5). The velocity gradients of these cloud ensembles are used to position them in the W43 region. Cloud ensemble \#1 may fall from the high-latitude part of the Galactic disk, \#2 seems to originate in the ScutumCentaurus arm, and \#3 may be at the very tip of the long bar. This picture is consistent with W43 being in front of the long bar, a region with crowded orbits known to be efficient in accumulating gas.

3. These three (mostly) molecular ensemble of clouds are separated by $\sim 20-40 \mathrm{~km} \mathrm{~s}^{-1}$ along the line of sight and converge on the location of W43. The measured velocity gradients extend within the W43 complex as velocity structures, are consistent with free-fall velocities, and display $\sim 20 \mathrm{~km} \mathrm{~s}^{-1} \mathrm{ve}-$ locity jumps where they merge. The cloud ensembles \#1-\#3 could thus be streams that are accreted onto the central W43 region, which has a total mass of $\sim 10^{7} M_{\odot}$, driven by gravity rather than forced by converging flows of atomic gas.

4. The HI surface density measured throughout the W43 complex is high when compared to the values usually quoted in the literature: $\Sigma_{\mathrm{HI}} \sim 65 \pm 20 M_{\odot} \mathrm{pc}^{-2}$ instead of 7-15 $M_{\odot} \mathrm{pc}^{-2}$. The H I surface density does not show a saturated behavior but instead increases when entering the molecular complex (see Fig. 7). This suggests that W43 is a heterogeneous structure of mixed $\mathrm{H}_{2}$ and $\mathrm{H} \mathrm{I}$ gas and that 
equilibrium between $\mathrm{H}_{2}$ formation and photodissociation is not reached. These results thus argue for a non steady-state formation of molecular clouds in W43.

5. The $\mathrm{H}_{2}$-to-H I ratio measured over the complete $\mathrm{W} 43$ region is high, $R_{\mathrm{H}_{2}} \sim 3.5 \pm_{2}^{3}$, proving that most of the gas is already in molecular form before reaching W43 (see Fig. 8). High-density regions of galaxies, such as the W43 molecular cloud complex, located in what was previously called the molecular ring of the Milky Way, obviously cannot provide constraints on the transition from atomic to molecular material. The formation of such molecular cloud entities is therefore weakly related to the efficiency of turning H I atoms into $\mathrm{H}_{2}$ molecules.

We conclude that the W43 molecular cloud complex most probably formed through a dynamical process such as the collision of several clouds, already in a molecular-dominated regime. The geometry and strength of this collision, as well as the molecular ratio and structure of the clouds, should be used to customize colliding cloud models. This is one of the goals of the W43-HERO IRAM large program, which uses the IRAM $30 \mathrm{~m}$ telescope to determine the diagnostics of colliding clouds and explain the extreme characteristics of the W43 molecular cloud star-forming region.

Acknowledgements. Part of this work was supported by the French National Agency for Research (ANR) projects "PROBeS" and "STARFICH", numbers ANR-08-BLAN-0241 and ANR-11-BS56-010. This project is carried out within the Collaborative Research Council 956, subproject A4, funded by the Deutsche Forschungsgemeinschaft (DFG). S.G. acknowledges support from the DFG via SFB project 881 "The Milky Way System" (sub-projects B1, B2 and B8). This publication made use of the synergy between observers and modelers set up for the large IRAM program W43-HERO. We are grateful to Snežana Stanimirović and Simon Bihr for useful discussions.

\section{References}

Anderson, L. D., \& Bania, T. M. 2009, ApJ, 690, 706

Andersson, B.-G., \& Wannier, P. G. 1993, ApJ, 402, 585

Andersson, B.-G., Roger, R. S., \& Wannier, P. G. 1992, A\&A, 260, 355

Athanassoula, E. 1992, MNRAS, 259, 345

Audit, E., \& Hennebelle, P. 2005, A\&A, 433, 1

Ballesteros-Paredes, J., Hartmann, L., \& Vázquez-Semadeni, E. 1999, ApJ, 527, 285

Banerjee, R., Vázquez-Semadeni, E., Hennebelle, P., \& Klessen, R. S. 2009, MNRAS, 398, 1082

Barriault, L., Joncas, G., Falgarone, E., et al. 2010, MNRAS, 406, 2713

Benjamin, R. A., Churchwell, E., Babler, B. L., et al. 2005, ApJ, 630, L149

Bigiel, F., Leroy, A., Walter, F., et al. 2008, AJ, 136, 2846

Blitz, L., \& Rosolowsky, E. 2006, ApJ, 650, 933

Blitz, L., \& Shu, F. H. 1980, ApJ, 238, 148

Blitz, L., \& Thaddeus, P. 1980, ApJ, 241, 676

Bloemen, J. B. G. M., Strong, A. W., Mayer-Hasselwander, H. A., et al. 1986, A\&A, 154, 25

Bournaud, F., Elmegreen, B. G., Teyssier, R., Block, D. L., \& Puerari, I. 2010, MNRAS, 409, 1088

Carlhoff, P., Nguyen Luong, Q., Schilke, P., et al. 2013, A\&A, 560, A24

Clark, P. C., Glover, S. C. O., Klessen, R. S., \& Bonnell, I. A. 2012, MNRAS, 424, 2599

Dame, T. M., \& Thaddeus, P. 2011, ApJ, 734, L24

Dame, T. M., Hartmann, D., \& Thaddeus, P. 2001, ApJ, 547, 792
Dib, S., Helou, G., Moore, T. J. T., Urquhart, J. S., \& Dariush, A. 2012, ApJ, 758,125

Dobbs, C. L. 2008, MNRAS, 391, 844

Dobbs, C. L., \& Pringle, J. E. 2013, MNRAS, 432, 653

Elmegreen, B. G., \& Elmegreen, D. M. 1987, ApJ, 320, 182

Glover, S. C. O., \& Mac Low, M.-M. 2007, ApJ, 659, 1317

Glover, S. C. O., Federrath, C., Mac Low, M.-M., \& Klessen, R. S. 2010, MNRAS, 404, 2

González-Fernández, C., \& Negueruela, I. 2012, A\&A, 539, A100

González-Fernández, C., López-Corredoira, M., Amôres, E. B., et al. 2012, A\&A, 546, A107

Hartmann, L., Ballesteros-Paredes, J., \& Bergin, E. A. 2001, ApJ, 562, 852

Hartmann, L., Ballesteros-Paredes, J., \& Heitsch, F. 2012, MNRAS, 420, 1457

Heitsch, F., \& Hartmann, L. 2008, ApJ, 689, 290

Heitsch, F., Burkert, A., Hartmann, L. W., Slyz, A. D., \& Devriendt, J. E. G. 2005, ApJ, 633, L113

Heitsch, F., Hartmann, L. W., Slyz, A. D., Devriendt, J. E. G., \& Burkert, A 2008, ApJ, 674, 316

Hennebelle, P., \& Pérault, M. 1999, A\&A, 351, 309

Hennebelle, P., Banerjee, R., Vázquez-Semadeni, E., Klessen, R. S., \& Audit, E. 2008, A\&A, 486, L43

Hennemann, M., Motte, F., Schneider, N., et al. 2012, A\&A, 543, L3

Hill, T., Motte, F., Didelon, P., et al. 2011, A\&A, 533, A94

Imara, N., \& Blitz, L. 2011, ApJ, 732, 78

Inoue, T., \& Inutsuka, S. 2012, ApJ, 759, 35

Jackson, J. M., Rathborne, J. M., Shah, R. Y., et al. 2006, ApJS, 163, 145

Koyama, H., \& Inutsuka, S. 2000, ApJ, 532, 980

Krumholz, M. R., McKee, C. F., \& Tumlinson, J. 2009, ApJ, 693, 216

Lee, M.-Y., Stanimirović, S., Douglas, K. A., et al. 2012, ApJ, 748, 75

López-Corredoira, M., Cabrera-Lavers, A., Mahoney, T. J., et al. 2007, AJ, 133 154

Louvet, F., Motte, F., Hennebelle, P., et al. 2014, A\&A, 570, A15

Mac Low, M.-M., \& Glover, S. C. O. 2012, ApJ, 746, 135

Martin, P., \& Friedli, D. 1997, A\&A, 326, 449

Molinari, S., Swinyard, B., Bally, J., et al. 2010, A\&A, 518, L100

Motte, F., Schilke, P., \& Lis, D. C. 2003, ApJ, 582, 277

Motte, F., Zavagno, A., Bontemps, S., et al. 2010, A\&A, 518, L77

Motte, F., Bontemps, S., Hennemann, M., et al. 2012, in SF2A-2012: Proc. Annual meeting of the French Society of Astronomy and Astrophysics, eds. S. Boissier, P. de Laverny, N. Nardetto, et al. 45

Negueruela, I., González-Fernández, C., Marco, A., Clark, J. S., \& Martínez-Núñez, S. 2010, A\&A, 513, A74

Negueruela, I., Marco, A., González-Fernández, C., et al. 2012, A\&A, 547, A15 Nguyen Luong, Q., Motte, F., Hennemann, M., et al. 2011a, A\&A, 535, A76

Nguyen Luong, Q., Motte, F., Schuller, F., et al. 2011b, A\&A, 529, A41

Nguyen-Luong, Q., Motte, F., Carlhoff, P., et al. 2013, ApJ, 775, 88

Renaud, F., Bournaud, F., Emsellem, E., et al. 2013, MNRAS, 436, 1836

Rodríguez-Fernández, N. J., \& Combes, F. 2008, A\&A, 489, 115

Roman-Duval, J., Jackson, J. M., Heyer, M., et al. 2009, ApJ, 699, 1153

Sawada, T., Hasegawa, T., \& Koda, J. 2012, ApJ, 759, L26

Shetty, R., Glover, S. C., Dullemond, C. P., et al. 2011, MNRAS, 415, 3253

Shibai, H., Okuda, H., Nakagawa, T., et al. 1991, ApJ, 374, 522

Spitzer, L. 1978, Physical processes in the interstellar medium (Wiley VCH)

Stil, J. M., Taylor, A. R., Dickey, J. M., et al. 2006, AJ, 132, 1158

Vallée, J. P. 2008, AJ, 135, 1301

van der Laan, T. P. R., Schinnerer, E., Boone, F., et al. 2011, A\&A, 529, A45

van Dishoeck, E. F., \& Black, J. H. 1986, ApJS, 62, 109

Vázquez-Semadeni, E., Passot, T., \& Pouquet, A. 1996, ApJ, 473, 881

Vázquez-Semadeni, E., Gómez, G. C., Jappsen, A. K., et al. 2007, ApJ, 657, 870

Vázquez-Semadeni, E., Colín, P., Gómez, G. C., Ballesteros-Paredes, J., \& Watson, A. W. 2010, ApJ, 715, 1302

Wannier, P. G., Lichten, S. M., \& Morris, M. 1983, ApJ, 268, 727

Williams, J. P., Blitz, L., \& Stark, A. A. 1995, ApJ, 451, 252

Wong, T., Hughes, A., Fukui, Y., et al. 2009, ApJ, 696, 370

Wozniak, H. 2007, A\&A, 465, L1

Zhang, B., Moscadelli, L., Sato, M., et al. 2014, ApJ, 781, 89 